\title{
MID-INFRARED EXTINCTION AND ITS VARIATION WITH GALACTIC LONGITUDE
}

\author{
JiAn GAO ${ }^{1,2}$, B. W. JiAng ${ }^{1}$, AND AIgen Li ${ }^{2}$ \\ ${ }^{1}$ Department of Astronomy, Beijing Normal University, Beijing 100875, China; jiangao@bnu.edu.cn, bjiang@bnu.edu.cn \\ 2 Department of Physics and Astronomy, University of Missouri, Columbia, MO 65211, USA; lia@ missouri.edu \\ Received 2009 May 4; accepted 2009 October 19; published 2009 November 18
}

\begin{abstract}
Based on the data obtained from the Spitzer/Galactic Legacy Infrared Midplane Survey Extraordinaire (GLIPMSE) Legacy Program and the Two Micron All Sky Survey (2MASS) project, we derive the extinction in the four IRAC bands, [3.6], [4.5], [5.8], and [8.0] $\mu \mathrm{m}$, relative to the 2MASS $K_{s}$ band (at $2.16 \mu \mathrm{m}$ ) for 131 GLIPMSE fields along the Galactic plane within $|l| \leqslant 65^{\circ}$, using red giants and red clump giants as tracers. As a whole, the mean extinction in the IRAC bands (normalized to the 2MASS $K_{s}$ band), $A_{[3.6]} / A_{K_{s}} \approx 0.63 \pm 0.01, A_{[4.5]} / A_{K_{s}} \approx 0.57 \pm 0.03$, $A_{[5.8]} / A_{K_{s}} \approx 0.49 \pm 0.03, A_{[8.0]} / A_{K_{s}} \approx 0.55 \pm 0.03$, exhibits little variation with wavelength (i.e., the extinction is somewhat flat or gray). This is consistent with previous studies and agrees with that predicted from the standard interstellar grain model for $R_{V}=5.5$ by Weingartner \& Draine. As far as individual sightline is concerned, however, the wavelength dependence of the mid-infrared interstellar extinction $A_{\lambda} / A_{K_{s}}$ varies from one sightline to another, suggesting that there may not exist a "universal" IR extinction law. We, for the first time, demonstrate the existence of systematic variations of extinction with Galactic longitude which appears to correlate with the locations of spiral arms as well as with the variation of the far-infrared luminosity of interstellar dust.
\end{abstract}

Key words: dust, extinction - Galaxy: bulge - Galaxy: structure - infrared: ISM - infrared: stars

Online-only material: color figures, machine-readable table

\section{INTRODUCTION}

With the development of space infrared (IR) astronomy, the precise determination of IR extinction becomes urgent in order to recover the intrinsic colors and spectral energy distributions (SEDs) of heavily obscured sources. There have been various attempts to measure the IR extinction based on the Infrared Space Observatory (ISO) and Spitzer Space Telescope since Lutz et al. (1996) obtained the mid-IR extinction from several hydrogen recombination lines and demonstrated the absence of the model-predicted pronounced minimum around $7 \mu \mathrm{m}$. This was supported by Jiang et al. $(2003,2006)$ based on the ISOGAL database (Omont et al. 1999), and by Indebetouw et al. (2005) based on the data from the Spitzer Galactic Legacy Infrared Midplane Survey Extraordinaire (GLIMPSE) Legacy Program (Benjamin et al. 2003). All these results roughly agree with the extinction predicted by the standard interstellar grain model for $R_{V}=5.5$ of Weingartner \& Draine (2001) and Draine (2003). ${ }^{3}$ However, so far only a few wave bands have been investigated and the sky coverage is also limited. No consensus has been reached yet regarding the interstellar extinction at $\sim 5-8 \mu \mathrm{m}$.

Recent progress in the IR extinction measurements is made toward star-forming regions mainly based on the Spitzer observations. Thanks to the high sensitivity of Spitzer, deep photometry is now possible and objects that suffer severe extinction are now reachable. Flaherty et al. (2007) studied five nearby star-forming regions at mid-IR wavelengths $(3.6 \mu \mathrm{m}, 4.5 \mu \mathrm{m}$, $5.8 \mu \mathrm{m}$, and $8.0 \mu \mathrm{m}$ from the InfraRed Array Camera [IRAC], ${ }^{4}$ and $24 \mu \mathrm{m}$ from the Multiband Imaging Photometer [MIPS]). They confirmed a relatively flat extinction curve at $\sim 4-8 \mu \mathrm{m}$. Román-Zúñiga et al. (2007) studied a star-forming dense cloud

\footnotetext{
$3 R_{V} \equiv A_{V} / E(B-V)$ is the total-to-selective extinction ratio, where $E(B-V) \equiv A_{B}-A_{V}$, the color excess, is the difference between the extinction in $B$ and $V$ bands.

4 The effective wavelengths of the four IRAC bands are actually $3.545 \mu \mathrm{m}$,
} $4.442 \mu \mathrm{m}, 5.675 \mu \mathrm{m}$ and $7.760 \mu \mathrm{m}$, respectively. core located in the Pipe Nebula, and found that the IR extinction in the IRAC bands of that region also agrees with the $R_{V}=5.5$ model curve and indicates a dust size distribution favoring larger sizes.

Although both the ISO and Spitzer measurements agree with each other in that the $\sim 5-8 \mu \mathrm{m}$ extinction is relatively flat and lacks the model-predicted minimum around $7 \mu \mathrm{m}$ of Draine (1989), there do exist differences among various measurements made for different sightlines. In their sample of five sightlines toward star-forming regions, Flaherty et al. (2007) found a clear difference between one sightline and the other four sightlines (see their Table 3). They derived higher $A_{\lambda} / A_{K_{s}}$ ratios and a flatter wavelength dependence than that of Indebetouw et al. (2005) for the same sightline toward $l=284^{\circ}$ in the Galactic plane.

From $>200$ fields observed in the ISOGAL survey, Jiang et al. (2006) analyzed the extinction at $7 \mu \mathrm{m}$ and $15 \mu \mathrm{m}$ along $\sim 120$ directions. They found marginal variation of the extinction at $7 \mu \mathrm{m}$. It is commonly believed that, with the parameter $R_{V}$ increasing in denser regions, the variation of the ultraviolet (UV) and visual extinction with wavelength becomes flatter than that of the diffuse interstellar medium (ISM) which is characterized with a lower $R_{V}$ (Cardelli et al. 1989), while the near-IR extinction seems to be "universal," with little variation among different sightlines (Draine 1989). However, Nishiyama et al. (2006a, 2009) recently argued against such a universal near-IR extinction. In addition, Fitzpatrick (2004) argued that the IRthrough-UV Galactic extinction curves should not be considered as a simple one-parameter family, whether characterized by $R_{V}$ as suggested by Cardelli et al. (1989) or any other parameters.

Whittet (1977) presented observational evidence for a small but appreciable variation in $R_{V}$ with Galactic longitude. He suggested that the most likely explanation for this is a variation in the mean size of the dust in the local spiral arm. However, unfortunately only a few data points were used in that work and therefore no systematic variation of the extinction with 
Galactic longitude was reported. Jiang et al. (2006) obtained the extinction around $7 \mu \mathrm{m}$ for 129 different sightlines, and no clear variation with Galactic longitude was found, although the extinction ratio $A_{[7]} / A_{K_{s}}$ does appear to exhibit a tendency of decreasing toward the Galactic center where $|l|<2^{\circ}$ (Jiang et al. 2006). The GLIMPSE Legacy Program surveyed the Galactic plane, with a large area coverage $\left(|l| \leqslant 65^{\circ}\right)$ and a detection limit of $\sim 15.5-13.0 \mathrm{mag}$ from 3.6 to $8.0 \mu \mathrm{m}$ (Churchwell et al. 2006). It provides an opportunity to explore the systematic variation of interstellar extinction in the IR with Galactic longitude.

In this work, we explore whether the mid-IR extinction varies among sightlines and how it varies in different interstellar environments based on the Spitzer/GLIMPSE database. In Section 2, the GLIMPSE data used in this work are briefly described. Section 3 presents the method adopted to derive the extinction. In Section 4, we discuss the selection of two different types of tracers (i.e., red giants and red clump giants). Section 5 reports the resulting extinction ratios $A_{\lambda} / A_{K_{s}}$ and the mean extinction from the total 131 GLIMPSE fields. Also discussed in Section 5 are the comparison of the extinction derived here with previous studies performed by Indebetouw et al. (2005) and Flaherty et al. (2007), and the longitudinal variation of the extinction ratios $A_{\lambda} / A_{K_{s}}$ as well as its relation with the Galactic spiral arms and the distribution of interstellar dust. In Section 6, we summarize our major conclusions.

\section{DATA: GLIMPSE AND 2MASS}

The data used in this work are obtained by the GLIMPSE group. GLIMPSE is a Spitzer Legacy Program to carry out an IR survey of the inner Galactic plane using the IRAC camera on board the Spitzer Space Telescope (Benjamin et al. 2003). It spans three cycles, GLIMPSE, GLIMPSE-II, and GLIMPSE3D (see Churchwell et al. 2009). These GLIMPSE programs observed a large part of the Galactic disk, including various sightlines along the Galactic plane and providing an opportunity to investigate whether the mid-IR extinction varies from one sightline to another.

The GLIMPSE- and GLIMPSE-II-enhanced data have now been released. ${ }^{5}$ These products consist of the highly reliable Point Source Catalogs (GLMC), the more complete Point Source Archives (GLMA), and mosaic images of the survey areas. In this study, we will use the GLMC catalogs since the sources in these catalogs were selected requiring the reliability to exceed $\sim 99.5 \%$ (Churchwell et al. 2009). Moreover, in the enhanced GLIMPSE version 2.0 catalogs and GLIMPSE-II version 1.0 catalogs, the point sources were band-merged (crossidentified) with the Two Micron All Sky Survey (2MASS) Point Source Catalog (see Cutri et al. 2003). They provide both magnitudes and fluxes in the four IRAC bands and three 2MASS bands.

Based on the original GLIMPSE and GLIMPSE-II Catalog files, we divided the Galactic plane into 131 fields. ${ }^{6}$ In this work, only the sources with a signal-to-noise ratio $\mathrm{S} / \mathrm{N} \geqslant 5$ in all three 2MASS bands and four IRAC bands are taken into account. More details about sample selection will be described in Section 4. Benefiting from the numerous detections by Spitzer/IRAC, the number of sources with $\mathrm{S} / \mathrm{N} \geqslant 5$ in a single

\footnotetext{
5 Data are available on http://www.astro.wisc.edu/sirtf/glimpsedata.html.

6 The fields overlapped by GLIMPSE and GLIMPSE-II are combined into one field, i.e., the fields of $l=9.0$ to $10^{\circ}$ and $l=350^{\circ}$ to $351^{\circ}$. Meanwhile, because of the insufficiency of sources, the two fields from $l=65^{\circ}$ to $65^{\circ} .3$ and $l=294.8$ to $295^{\circ}$ are incorporated into the fields $l=64^{\circ}$ to $65^{\circ}$ and $l=295^{\circ}$ to $296^{\circ}$, respectively.
}

sky field exceeds 10,000 for almost all the GLIMPSE fields. The only exception is the GLIMPSE Observation Strategy Validation (OSV) field, with $l=283^{\circ} .8$ to 284.6 and $|b|<1^{\circ}$, where the number of sources with $S / N \geqslant 5$ is 9625 .

\section{METHOD}

\subsection{Color-excess Method}

The determination of dust extinction is most commonly made by comparing the flux densities of extincted and unextincted pairs of stars of the same spectral type (Draine 2003). Lutz (1999) used the $\mathrm{H}$ recombination lines detected between 2.5 and $9 \mu \mathrm{m}$ to probe the extinction law in this wavelength range of the Galactic center based on a comparison of the observed line fluxes with that expected from the standard Case B recombination. Here we adopt the "color-excess" method to obtain the extinction. This method calculates the ratio of two color excesses which can be expressed as follows:

$$
\begin{aligned}
k_{x} \equiv \frac{E\left(\lambda_{r}-\lambda_{x}\right)}{E\left(\lambda_{c}-\lambda_{r}\right)} & =\frac{\left(\lambda_{r}-\lambda_{x}\right)_{\text {observed }}-\left(\lambda_{r}-\lambda_{x}\right)_{\text {intrinsic }}}{\left(\lambda_{c}-\lambda_{r}\right)_{\text {observed }}-\left(\lambda_{c}-\lambda_{r}\right)_{\text {intrinsic }}} \\
& =\frac{A_{r}-A_{x}}{A_{c}-A_{r}},
\end{aligned}
$$

where $\lambda_{x}$ is the magnitude in the band $x$ of interest, $\lambda_{r}$ is the magnitude in the reference band $r$ (which is usually taken to be the $\mathrm{K}$ or $K_{s}$ band), and $\lambda_{c}$ is magnitude in the comparison band $c$ (which is usually taken to be the $J$ or $H$ band). Therefore, the extinction ratio of the $x$ band to the $r$ reference band is

$$
A_{x} / A_{r}=1+k_{x}\left(1-A_{c} / A_{r}\right) .
$$

Note that $A_{c} / A_{r}$ is always greater than 1 (no matter whether the $J$ band or the $H$ band is chosen as the comparison band), since it is generally true that $A_{J}, A_{H}>A_{K}, A_{K_{s}}$. Therefore $A_{x} / A_{r}$ increases with the decreasing of $k_{x}$.

The "color-excess" method is widely applied to photometric data and can probe deeper than the spectrum-pair method. Most of the IR extinction determination studies are performed using this method. In the color-excess method, a group of sources that have the same intrinsic color indices $\left(\lambda_{r}-\lambda_{x}\right)_{\text {intrinsic }}$ and $\left(\lambda_{c}-\lambda_{r}\right)_{\text {intrinsic }}$ are chosen, $k_{x}$ is simply the slope of the line that linearly fits the observed color indices $\left(\lambda_{r}-\lambda_{x}\right)_{\text {observed }}$ and $\left(\lambda_{c}-\lambda_{r}\right)_{\text {observed }}$ (Jiang et al. 2003). This is a statistical method as it makes use of a large number of sources and reduces the risk of depending on any individual objects with large uncertainties in the determination of their intrinsic color indices. Meanwhile, it is essential for the accuracy of the method to have a homogeneous sample (i.e., with very small scatter in the color indices $\left(\lambda_{r}-\lambda_{x}\right)_{\text {intrinsic }}$ and $\left.\left(\lambda_{c}-\lambda_{r}\right)_{\text {intrinsic }}\right)$.

\subsection{Comparison Bands: $J$ and $H$}

From Equations (1) and (2), it can be seen that the determination of $A_{x} / A_{r}$, the ratio of the $x$-band extinction to the extinction of the reference band $r$, requires the knowledge of $A_{c} / A_{r}$, the ratio of extinction at the comparison band $c$ to that at the reference band $r$. In previous studies, both the $J$ (Indebetouw et al. 2005; Jiang et al. 2003, 2006; Román-Zúñiga et al. 2007) and the $H$ bands (Flaherty et al. 2007) have been used as the comparison band. The advantage of taking the $J$ band as the comparison band is that $E\left(J-K_{s}\right)$ is more sensitive to the extinction as $E\left(J-K_{s}\right)$ is about twice $E\left(H-K_{s}\right)$ for the 
same value of $A_{K_{s}}$ (the extinction at the $K_{s}$ band). The advantage of choosing the $H$ band as the comparison band is that there are more red giants detected in the $H$ band than in the $J$ band. This would be particularly important when the sample size is small which could influence the statistics. But for the sky fields studied here, the sample size is not a problem thanks to the sensitivity of Spitzer/IRAC. In this work, we therefore adopt the $J$ band as the comparison band. The $J$ band was also selected as the comparison band in our previous studies of the ISOGAL fields (Jiang et al. 2003, 2006). But in order to compare with the results of Flaherty et al. (2007), we also calculate $A_{x} / A_{r}$ with the $H$ band taken as the comparison band.

We take $A_{J} / A_{K_{s}}=2.52$ (with $J$ as the comparison band) or $A_{H} / A_{K_{s}}=1.56$ (with $H$ as the comparison band) as derived by Rieke \& Lebofsky (1985) for sightlines toward the GC. ${ }^{7}$

\section{TRACERS: RED GIANTS AND RED CLUMP STARS}

\subsection{Red Giants}

In the IR, red giants are appropriate tracers of interstellar extinction ${ }^{8}$ for the following reasons: (1) they have a narrow range of effective temperatures so that the scatter of the intrinsic color indices is small. The $J-K_{s}$ color index, often chosen to describe $\lambda_{c}-\lambda_{r}$, has a scatter of only $\sim 0.1$ mag around the central value of $\sim 1.2$; (2) they are bright in the IR and remain visible even with large extinction and/or at a great distance. ${ }^{9}$ On average, their absolute $K_{s}$ magnitudes are as bright as $M_{K_{s}} \sim-5.0 \mathrm{mag}$, making them readily detectable by 2 MASS even at a distance to the GC $(\sim 8.5 \mathrm{kpc})$. But we should note that evolved red giants may have a circumstellar dust shell which would cause circumstellar extinction and produce IR emission, affecting our understanding of their intrinsic color indices. Accordingly, the selection of red giants is usually based on the mid-IR colors which are barely affected by interstellar extinction. Following Jiang et al. (2003, 2006), we adopt the following criteria to select red giants as our samples.

1. [3.6] $-[4.5]<0.6$ and [5.8] $-[8.0]<0.2$, also adopted by Flaherty et al. (2007). We confine ourselves to colors bluer than certain values to exclude the sources with IR excess such as pre-main-sequence stars and asymptotic giant branch (AGB) stars. This criterion also safely excludes young stellar objects (YSOs; Allen et al. 2004; Megeath et al. 2004). However, it is more complicated for evolved stars. By analyzing the synthetic colors of AGB stars obtained by convolving the ISO Short Wavelength Spectrometer (ISOSWS) spectra with the IRAC transmission profiles, Marengo et al. (2007) found that the IRAC colors of AGB stars are similar to that of red giant branch (RGB) stars. Therefore, the selected samples may be contaminated by some AGB stars. Groenewegen (2006) also

\footnotetext{
7 Indebetouw et al. (2005) estimated $A_{J} / A_{K_{S}} \approx 2.5 \pm 0.2$ and $A_{H} / A_{K_{s}} \approx 1.55 \pm 0.1$ for the $l=42^{\circ}$ and $284^{\circ}$ sightlines in the Galactic plane which are very close to that of Rieke \& Lebofsky (1985). However, Nishiyama et al. (2006a) derived $A_{J} / A_{K_{S}} \approx 3.02$ and $A_{H} / A_{K_{S}} \approx 1.73$ for the sightlines toward the GC based on red clump stars. They found a steep power law for the near-IR extinction $\left(A_{\lambda} \propto \lambda^{-1.99}\right)$, while in literature it is often thought that the near-IR extinction is a "universal" power law $A_{\lambda} \propto \lambda^{-\beta}$ with $\beta \approx 1.6-1.8$ (Draine 1989, 2003).

8 Red giants were used as tracers to derive the extinction at $7 \mu \mathrm{m}$ and $15 \mu \mathrm{m}$ by Jiang et al. (2003, 2006). The results were in close agreement with that from the hydrogen recombination lines (Lutz et al. 1996; Lutz 1999).

9 With $M_{K_{s}} \sim-5.0 \mathrm{mag}$ and $m_{K_{s}} \sim 13 \mathrm{mag}$, the distance can be as large as $d \approx 40 \mathrm{kpc}$, provided there is no extinction along the line of sight toward to the star.
}

found [3.6] $-[4.5]>0.3$ for AGB stars with a significant mass loss. The [5.8] - [8.0] color index is slightly more selective, with young AGB stars being redder by $\sim 0.2$ than most red giants. Nevertheless, the criteria are kept with significant confidence as red giants are much more numerous than AGB stars in these relatively blue colors. This is later proved to be correct in the appearance of the color-color diagram of the sources. But it should be kept in mind that some AGB stars that suffer circumstellar extinction may be included. $^{10}$

2. $J-K_{s}>1.2$ or $H-K_{s}>0.3$. This criterion excludes foreground dwarf stars since even late-M dwarfs have $J-K_{s}<0.6$. Both theoretical and observational studies suggest that RGB stars have an intrinsic color of $J-K_{s} \approx$ 1.2 magwith a dispersion of $\sim 0.1 \mathrm{mag}$ (Glass et al. 1999; Bertelli et al. 1994). For an M5 giant, the calculated $J-K_{s}$ is $\sim 1.2 \mathrm{mag}$, and $H-K_{s}$ is $\sim 0.3 \mathrm{mag} .{ }^{11}$ In the following, the samples selected in terms of $J-K_{s}>1.2$ are denoted by RG1, while the ones selected from $H-K_{s}>0.3$ are referred as RG2.

3. Good photometric quality. This criterion guarantees the reliability of the calculated color indices without reducing the number of sources too much so as to degrade the statistical accuracy. Specifically, the photometry quality flags in the 2MASS $J H K_{s}$ bands are "AAA" (i.e., with $\mathrm{S} / \mathrm{N} \geqslant 5)$; the quality flags in the IRAC bands are comparable (in the GLIMPSE catalogs the photometric uncertainty is typically $<0.2 \mathrm{mag}$ ). For all the 131 sky fields studied here, $\mathrm{S} / \mathrm{N} \geqslant 5$ is required in all three $2 \mathrm{MASS}$ bands and four IRAC bands.

4. A deviation of $<3 \sigma$ from the line fitted to the observed colors $K_{s}-\lambda$ ( $\lambda$ is the IRAC band wavelength) versus $J-$ $K_{s}$. We used the IDL robust fitting program to iterate the fit, rejecting the sources with a deviation larger than $3 \sigma$. Those sources (with a deviation larger than $3 \sigma$ ) were rejected because, from a statistical point of view, it is unlikely for them to follow the linear relation. Furthermore, most of them have relatively large $K_{s}-\lambda$ values, indicating the possible presence of extinction arising from circumstellar envelopes (Jiang et al. 2006). Indebetouw et al. (2005) also rejected high- $\sigma$ points to exclude some of the extreme red excess sources.

Figure 1 shows the near-IR color-magnitude diagrams (CMDs) of two typical fields, $l=309^{\circ}-310^{\circ}$ and $l=11^{\circ}-12^{\circ}$. In the left panels (a, c), the RG1 samples (based on $J-K_{s}>1.2$ and the other criteria described above) are denoted by red dots. The black background points are the sources in those fields with $\mathrm{S} / \mathrm{N} \geqslant 1$ in all three 2MASS bands. Because we only adopt the sources with $\mathrm{S} / \mathrm{N} \geqslant 5$ in all seven bands (three 2MASS bands and four IRAC bands), the number of the selected RG samples is much smaller than the total number of sources in these fields.

\footnotetext{
10 We do not know the exact fraction of stars in these colors that are contaminated by AGB stars. But considering a star of 1.5 solar mass, it spends about $7.6 \times 10^{8} \mathrm{yr}$ at RGB and $\sim 10^{7} \mathrm{yr}$ at AGB (Vassiliadis \& Wood 1993). The number ratio of RGB/AGB would be about two orders of magnitude. In addition, their color index [5.8] - [8.0] of AGB stars ranges from about 0.0 to 1.0 (Marengo et al. 2008), mostly much redder than our critical value 0.2. Combining these two factors, it is a reasonable estimation that the fraction of AGB stars in the selected sample should be less than $1 \%$.

11 If the $H$ band is chosen as the comparison band, this criterion $\left(H-K_{s}>0.3\right)$ is comparable to that of Flaherty et al. (2007), i.e., $H-K_{s}>0.2$.
} 


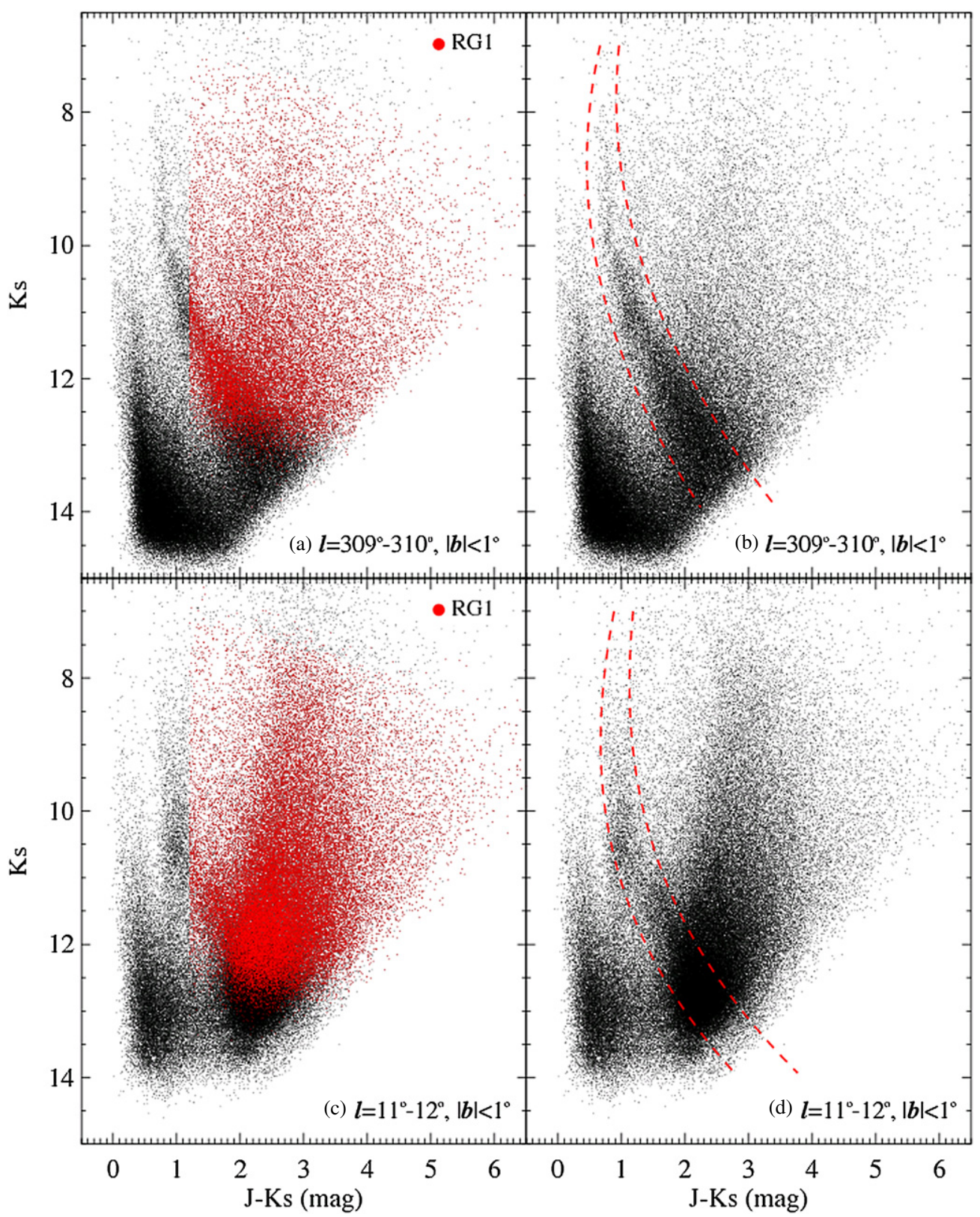

Figure 1. Color-magnitude diagrams for two GLIMPSE fields: $l=309^{\circ}-310^{\circ}$ and $|b|<1^{\circ}$ (upper panels: a, b), and $l=11^{\circ}-12^{\circ}$ and $|b|<1^{\circ}$ (lower panels: (c), (d)). Denoted by black dots, the sources in these fields with $\mathrm{S} / \mathrm{N} \geqslant 1$ in all three $2 \mathrm{MASS}$ bands are all plotted. In the left panels ((a), (c)), red dots denotes the red giants selected satisfying $J-K_{s}>1.2$ (RG1), $\mathrm{S} / \mathrm{N} \geqslant 5$ and the other criteria (see Section 4.1). In the right panels ((b), (d)), the selected RCG stars lie in between the two red dashed lines, with the center characterized by $\left(J-K_{s}\right)=5.28-1.11 K_{s}+0.07 K_{s}^{2}$ and $\left(J-K_{s}\right)=7.09-1.44 K_{s}+0.08 K_{s}{ }^{2}$ for $l=309^{\circ}-310^{\circ}$ and $l=11^{\circ}-12^{\circ}$, respectively. The half-widths of the RCG stripes are $\sim 0.3$ mag at $K_{s}=13$ for panel (b), $\sim 0.2 \mathrm{mag}$ at $K_{s}=13$ for panel (d).

(A color version of this figure is available in the online journal.)

\subsection{Red Clump Giants}

\subsubsection{Red Clump Giants as a Tracer}

Indebetouw et al. (2005) chose red clump giants (RCGs), which have a dispersion of $\sim 0.3 \mathrm{mag}$ in the absolute $K_{s}$ magnitude and $\sim 0.2 \mathrm{mag}$ in the near-IR color index (LópezCorredoira et al. 2002), as a tracer to derive the extinction from the 2MASS $J$ band to the IRAC $8.0 \mu \mathrm{m}$ band. RCG stars are the equivalent of the horizontal-branch stars for a metalrich population. They have narrow distributions in luminosity and color indices, with a weak dependence on metallicity (Nishiyama et al. 2006a). Thus, their locations in the near-IR CMDs could be more clearly distinguished than those of red giants. This makes them a more homogeneous sample of tracers to obtain the IR extinction. ${ }^{12}$

However, RCG stars are fainter than red giants in the IR because of their relatively blue color index (usually K-type) and

\footnotetext{
${ }^{12}$ RCG stars have long been used as a reliable standard candle to study the Galactic structure (e.g., see Demers et al. 1994; López-Corredoira et al. 2002; Nishiyama et al. 2006b), as the reddening along the line of sight toward RCG stars is commonly considered to be almost completely due to interstellar extinction. Precisely speaking, from the view of the evolutionary stage of RCG stars, they should be at the post-RGB phase, and small amounts of dust should be there arising from the small mass loss at the RGB phase. But this would not affect our results since they have very similar color indices (this earns them the name "clump"). Therefore, it is secure to attribute any excess color reddening to interstellar extinction.
} 
lower absolute luminosity $\left(M_{K_{s}} \sim-1.65 \mathrm{mag}\right.$, see Wainscoat et al. 1992; or $M_{K_{s}} \sim-1.61 \pm 0.01 \mathrm{mag}$, see Alves 2000). The RCG stars detected by 2MASS are not as distant as red giants. Therefore, they cannot trace the extinction as deep as red giants. Due to the weakness of the IR extinction, the traceable depth of extinction is crucial. As shown in Indebetouw et al. (2005), the maximum $E\left(J-K_{s}\right)$ traced by RCG stars was about 2.6 mag, much smaller than the easily reached 6 mag of red giants. In addition, the color dispersion of RCG stars, $\sim 0.2 \mathrm{mag}$, is a little bit larger than that of red giants, $\sim 0.1$ mag (Glass et al. 1999; van Loon et al. 2003). Nevertheless, in this work, RCG stars are also selected as a tracer in deriving the IR extinction, and the results are compared with those from the red giant samples.

As K2III giants, the commonly accepted absolute magnitude for RCG stars in the $K_{s}$ band is $M_{K_{s}} \approx-1.65 \mathrm{mag}$, and their intrinsic IR color indices are almost constant, with $\left(J-K_{s}\right) \approx$ 0.75 (Wainscoat et al. 1992). These characteristics would place them in a well-defined narrow stripe in the near-IR CMDs ( $J-K_{s}$ versus $K_{s}$ ). However, the distribution of RCG stars in the CMD (based on their observed magnitudes and colors) is much more scattered. This is because the observed color indices depend only on interstellar extinction, while the observed $K_{S}$ magnitudes depend not only on the amount of extinction but also on the distance of the star. In addition, the extinction is highly uneven and is not simply proportional to the distance. Furthermore, there might be contamination of dwarf stars and AGBs at the lower end in the CMDs. ${ }^{13}$ Thus, the selection of the RCG stars appears empirical and eye dependent.

In Figure 1, the right panels show the RCG stripes lying between two red dashed lines. The extension of the color index $\left(J-K_{s}\right)$ of red giants (see the left panels of Figure 1) clearly connects to that of red clump stars. The upper right CMD (Figure 1(b)) has a relatively clear branch of red clump stars, but this is not the case for the bottom one. In comparison with the quite scattered distribution of red giants, the disk RCG stars stand out by their configured locations in the CMDs for a large portion of the sightlines (e.g., the field $l=309^{\circ}-310^{\circ}$ ), i.e., a narrow stripe from upper-left to lower-right in the CMDs caused by interstellar extinction and the increasing distance from the Sun. Within the RCG strip, the red clump stars consist of relatively homogenous samples. However, the homogeneity depends very much on the specific field (e.g., the field with $l=11^{\circ}-12^{\circ}$ and $|b| \leqslant 1^{\circ}$ does not present a clear stripe of RCG stars in Figure 1(d)). The interstellar extinction is possibly highly nonuniform with distance on the sightline within $l=11^{\circ}-12^{\circ}$, so that a gap appears in the RCG stripe near $\left(J-K_{s}\right) \simeq 1.5$ and $K_{s} \simeq 11$. In addition, a giant branch can be seen at $J-K_{s} \approx 2.8$ in the lower CMD in Figure 1(d), which is probably due to the stars in the bulge as suggested by Hammersley et al. (2000).

Although the 2MASS Point Source Catalog is complete down to $K_{s} \approx 14.3 \mathrm{mag}$ (Cutri et al. 2003), the faintest $K_{s}$ magnitude in this work is $\sim 13 \mathrm{mag}$ for almost the entire GLIMPSE fields (because of the $\mathrm{S} / \mathrm{N} \geqslant 5$ requirement for all the 2MASS and IRAC bands). The faintest $K_{s}$ magnitude of the selected RG samples can only reach $\sim 13$ mag (see Figures 1 (a), (c)). For the RCG stars selected from the RCG stripes requiring $S / N \geqslant 5$ in all seven bands, the faintest $K_{S}$ magnitude can also only reach $\sim 13$ mag.

\footnotetext{
13 López-Corredoira et al. (2002) found that for $K_{s}<12.5$ only $\sim 2.5 \%-5 \%$ of the detected sources are dwarfs, but this fraction rises to $\sim 10 \%-40 \%$ for $13<K_{s}<14$ (López-Corredoira et al. 2002; Cabrera-Lavers et al. 2007).
}

\subsubsection{Selection of RCG Stars}

López-Corredoira et al. (2002) proposed a method to extract RCG stars based on CMDs. They determined an empirical track of RCG stars by linking the peaks of the histograms of all the horizontal cuts in the CMDs. Applying a limitation of $K_{s}<13.0$ to exclude $\mathrm{K}$ dwarf stars, the sources within a deviation of $<0.2$ mag in $J-K_{s}$ from the determined track are extracted as the selected RCG stars. Drimmel et al. (2003) and Indebetouw et al. (2005) adopted similar methods to pick up RCG stars. In this work, we take a similar approach.

First, a rough stripe in the $J-K_{s}$ versus $K_{s}$ CMD was chosen by eye to encircle the preliminary range of the RCG stars. The eye-selected range is divided into different horizontal cuts with a step of $0.3 \mathrm{mag}$ at $K_{s}$, then the histograms of each horizontal cut were fitted with Gaussian functions to determine the peak color indices $J-K_{s}$ for different horizontal $K_{s}$ cuts. Second, the peak positions in each horizontal cuts are taken as input to delineate the curve fitted with a second-order polynomial for the central location of the RCG stars. ${ }^{14}$ Third, the width of the curve derived from the previous step, which corresponds to the scale of the dispersion of the color index, is determined. Unlike López-Corredoira et al. (2002), we do not treat this width as a constant (because it results not only from the almost constant scatter of the intrinsic color index, but also from the photometric error which increases with decreasing apparent brightness, i.e., increasing magnitude). The increase of dispersion width with the observed magnitude is noticeable from the CMDs as well. So the width adopted in our work differs for different sightlines based on the definition of the RCG stripes in the CMDs. Centered with the fitted red clump track, the width ranges from $\sim 0.1$ to $\sim 0.2 \mathrm{mag}$ for $K_{s}=8.0$ and increases to $\sim 0.2-0.3 \mathrm{mag}$ for $K_{s}=13.0$ (see Figure 1)

Finally, one more factor to take into account is the enhanced contamination of dwarf stars at fainter magnitudes. These dwarfs should be mainly earlier than K-type to be bright enough to be visible at relatively large distances (because their observed colors are much redder than the intrinsic ones which must result from subjecting to substantial extinction, indicating that they cannot be nearby). The near-IR intrinsic colors of these dwarf stars are bluer than those of the RCG stars. Compared with the RCG stars in the mid-IR, these dwarfs would have a larger $k_{x}$ in Equation (1) and lead to a smaller $A_{x} / A_{r}$ in Equation (2) since $A_{c} / A_{r}>1$. To suppress this effect, the $K_{s}$ magnitude is limited to 13.0 in López-Corredoira et al. (2002). We also place an upper limit on the $K_{s}$ magnitude in order to reduce the confusion from the $\mathrm{K}$ dwarfs ${ }^{15}$ (but this upper limit is again different for different sightlines). As mentioned earlier, the $K_{S}$ magnitude can only reach $\sim 13$ mag for most of the GLIMPSE fields because of the $S / N \geqslant 5$ requirement for all seven bands (see Section 4.2.1). Consequently, for most of the sky fields, the typical limit on the $K_{s}$ magnitude is $\sim 12 \mathrm{mag}$. But this limit is much smaller on the sightlines toward the Galactic bulge direction, mostly in the range of $|l|<15^{\circ}$, where $K_{s} \leqslant 11.5$ is generally taken. The lower cut of the $K_{s}$ magnitude results in much smaller $J-K_{s}$ and much fewer samples than that from the red giant samples. Furthermore, because of the lower $K_{s}$ magnitude $\left(K_{s} \leqslant 12\right)$, the RCG samples in this work can only

\footnotetext{
14 Please contact the authors for the parameters if one is interested in carrying out similar computations.

15 To suppress the contaminations of YSOs and AGB stars, we adopt the same criterion on the IRAC color index as we did to select the RG samples, i.e., $[3.6]-[4.5]<0.6$ and $[5.8]-[8.0]<0.2$ (see Section 4.1).
} 
Table 1

Average Extinction (Relative to $A_{K_{s}}$ ) Over All 131 GLIMPSE Fields by Different Tracers

\begin{tabular}{lcccc}
\hline \hline \multicolumn{1}{c}{ Tracers } & $A_{[3.6]} / A_{K_{s}}$ & $A_{[4.5]} / A_{K_{s}}$ & $A_{[5.8]} / A_{K_{s}}$ & $A_{[8.0]} / A_{K_{s}}$ \\
\hline Red giants (RG1, $J)$ & $0.64 \pm 0.01$ & $0.63 \pm 0.01$ & $0.52 \pm 0.01$ & $0.55 \pm 0.02$ \\
Red giants (RG2, $J$ ) & $0.65 \pm 0.01$ & $0.63 \pm 0.02$ & $0.53 \pm 0.01$ & $0.55 \pm 0.02$ \\
Red giants (RG2, H) & $0.61 \pm 0.01$ & $0.59 \pm 0.02$ & $0.48 \pm 0.01$ & $0.50 \pm 0.02$ \\
Red clump giants (RCG, $J)_{\text {Mean }^{\text {a }}}$ & $0.61 \pm 0.01$ & $0.51 \pm 0.04$ & $0.45 \pm 0.04$ & $0.55 \pm 0.04$ \\
Nishiyama09 $^{\mathrm{b}}$ & $0.63 \pm 0.01$ & $0.57 \pm 0.03$ & $0.49 \pm 0.03$ & $0.55 \pm 0.03$ \\
Flaherty07c $^{\text {Indebetouw05 }}$ & $0.50 \pm 0.01$ & $0.39 \pm 0.01$ & $0.36 \pm 0.01$ & $0.43 \pm 0.01$ \\
& $0.631 \pm 0.005$ & $0.53 \pm 0.01$ & $0.48 \pm 0.01$ & $0.49 \pm 0.01$ \\
\hline
\end{tabular}

Notes.

${ }^{a}$ The mean extinction is calculated from the RG1 and RCG results.

${ }^{\mathrm{b}}$ Extinction toward the GC (Nishiyama et al. 2009).

${ }^{c}$ These are averaged results over five star-forming regions (Flaherty et al. 2007).

d The uncertainties on $A_{x} / A_{K_{s}}$ of Indebetouw et al. (2005) include the uncertainty of $A_{H} / A_{K_{s}}$.

be used to probe the interstellar extinction from the diffuse ISM near the Sun. ${ }^{16}$ According to the following expression:

$$
5 \log d=m_{K_{s}}-M_{K_{s}}+5-A_{K_{s}}(d)
$$

if $A_{K_{s}} \approx 1$, the limitation of $K_{s} \leqslant 12$ implies that the most distant stars that could be selected in our samples are at $d \sim 3.5 \mathrm{kpc}$. Many works have suggested the existence of a linear bar across the Galactic center with a half-length of $\sim 4.5 \mathrm{kpc}$ and a position angle of $\sim 45^{\circ}$ (Hammersley et al. 1994, 2000; Benjamin et al. 2005; Cabrera-Lavers et al. 2007), i.e., the closest distance to the Sun is $\sim 5.3 \mathrm{kpc}$ (assuming the distance to the GC from the sun is $R_{\text {sun }} \approx 8.5 \mathrm{kpc}$ ). Thus even if $A_{K_{s}} \approx 0$, we can still hardly reach the bulge stars located in the bar beyond $d \sim 5 \mathrm{kpc}$. In the region within $|l|<15^{\circ}$, where $K_{s} \leqslant 11.5$ is taken, the distance limit must be closer than $\sim 4 \mathrm{kpc}$. So the interstellar extinction derived from the RCG samples is mainly from the local diffuse ISM, and it may differ from that derived from red giants (see Section 5.2.2).

For most of the 131 GLIMPSE fields, the stripes of the RCG stars in the near-IR CMDs are clearly visible, indicating that the above-described selection method for RCG stars is reasonably reliable. But for the fields in the directions to the Galactic bulge, there are so many dwarf stars that the stripes of RCG stars are not easily distinguishable; thus, only the nearby RCG stars subject to small extinction which lie at the higher end of the stripes can be extracted. Moreover, in the regions within $|l|<15^{\circ}$, there is always a giant branch overlapping with the RCG stripe at the low $K_{s}$ end (see the vertical branch near $\left(J-K_{s}\right)=2.8$ in Figure 1(d)). Significant contamination occurs at later-type RGB and AGB stars (Hammersley et al. 2000; Indebetouw et al. 2005). The contamination caused by other types of stars degrades the quality of the results derived from the RCG samples. The reliability of the results varies from field to field.

\section{RESULTS AND DISCUSSION}

\subsection{Mean Extinction}

We have obtained the extinction in each IRAC band (relative to that of the $K_{s}$ band) averaged over all 131 GLIMPSE fields

\footnotetext{
16 The RCG stars in the Galactic bulge $\left(|l|<20^{\circ}\right)$ appear to lie at a larger distance (Nishiyama et al. 2006a) and have a $K_{s}$ magnitude of $\sim 12-14$ (Hammersley et al. 2000). Because of the $K_{s}$ magnitude limitation, the RCG stars finally extracted in this work would mostly lie in the Galactic disk.
}

(see Table 1). With red giants as the extinction tracer and the $J$ band as the comparison band, the mean extinction ratios are $A_{[3.6]} / A_{K_{s}} \approx 0.64 \pm 0.01, A_{[4.5]} / A_{K_{s}} \approx 0.63 \pm 0.01$, $A_{[5.8]} / A_{K_{s}} \approx 0.52 \pm 0.01$, and $A_{[8.0]} / A_{K_{s}} \approx 0.55 \pm 0.02$. The selection criteria of $J-K_{s}>1.2(\mathrm{RG} 1)$ or $H-K_{s}>0.3(\mathrm{RG} 2)$ make little difference since the samples selected from these two criteria are almost the same (see Figure 2 and the first two rows of Table 1). Here we adopt $A_{H} / A_{K_{s}}=1.56$ and $A_{J} / A_{K_{s}}=2.52$ (i.e., we take the power index $\beta$ of the near-IR extinction law $A_{\lambda} \propto \lambda^{-\beta}$ to be 1.61 ; see Rieke \& Lebofsky 1985; Draine 2003). With the $H$ band chosen as the comparison band, the extinction ratios $A_{\lambda} / A_{K_{s}}$ (derived from the RG2 samples, see the third row of Table 1) systematically decrease by an amount of $\sim 0.04$ at $3.6 \mu \mathrm{m}, \sim 0.04$ at $4.5 \mu \mathrm{m}, \sim 0.05$ at $5.8 \mu \mathrm{m}$, and $\sim 0.05$ at $8.0 \mu \mathrm{m}$, respectively. If we take a larger power index (e.g., $\beta=1.75$ as suggested by Draine (1989)), the extinction ratios will become smaller. ${ }^{17}$

Also shown in Table 1 are the extinction ratios $A_{\lambda} / A_{K_{s}}$ derived from the RCG samples (with the $J$ band as the comparison band). The extinction ratios of the $3.6 \mu \mathrm{m}, 4.5 \mu \mathrm{m}$, and $5.8 \mu \mathrm{m}$ bands derived based on the RCG samples are smaller than those based on red giants, while the extinction ratio of the $8 \mu \mathrm{m}$ band is approximately equal for both samples. ${ }^{18}$ As mentioned earlier, the RCG samples may include some dwarf stars at the faint end. Since dwarf stars are intrinsically bluer than RCG stars, they may cause the derived extinction to be underestimated (see Equation (2)). On the other hand, the extinction would be somewhat overestimated from the red giant samples because of their circumstellar dust. But for the $8.0 \mu \mathrm{m}$ band, the extinction derived from red giants may be underestimated because of the possible presence of silicate emission. ${ }^{19}$

Both Indebetouw et al. (2005) and Flaherty et al. (2007) have studied the extinction in the GLIMPSE OSV field. To allow a direct comparison with their results, in Table 2 we tabulate the IRAC band extinction (relative to $A_{K_{s}}$ ) derived here as well

\footnotetext{
17 This is true no matter which band, $J$ or $H$, is chosen as the comparison band. But the effect is more significant for the former and therefore there will be little difference between the extinction ratios derived using different comparison bands $(J$ or $H$ ).

18 We should note that the approximate equality between the extinction from the red giant and RCG samples in the $8.0 \mu \mathrm{m}$ band is an average result. As far as individual regions are concerned, the difference in this band is significant in the Galactic bulge region (see Section 5.2).

19 The red giants (mostly M-type stars) selected here may have a very thin shell of silicate dust and therefore it is likely that they exhibit a weak $9.7 \mu \mathrm{m}$ silicate emission feature. The uncertainties discussed here are not included in the quoted errors in Table 1.
} 


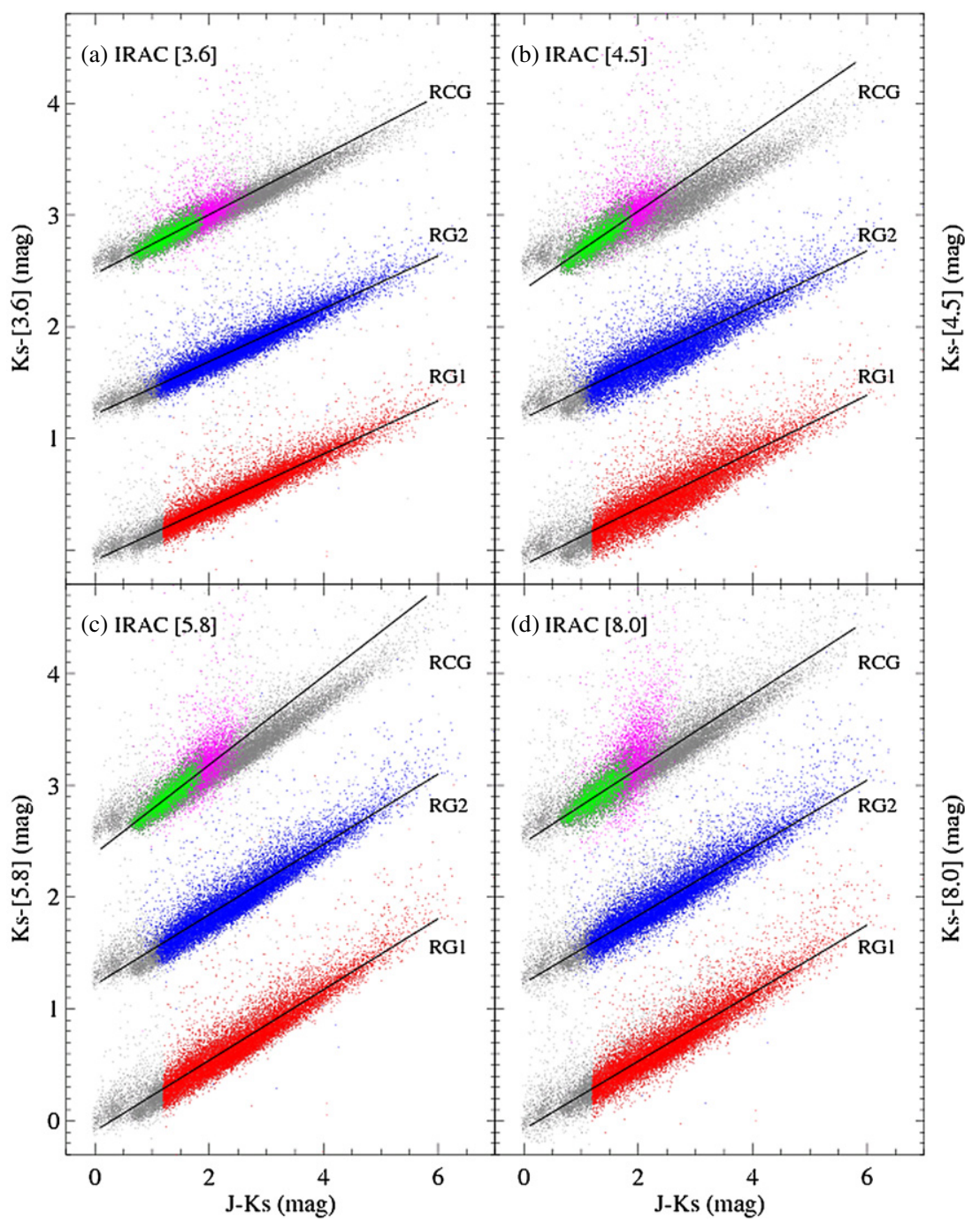

Figure 2. Color-color diagrams for the red giants (RG1, RG2) and red clump giants (RCG) selected in a typical sightline, with $l=309^{\circ}-310^{\circ}$ and $|b|<1^{\circ}$. For clarity, the RG2 and RGC samples are vertically shifted $+1.3 \mathrm{mag}$ and $+2.6 \mathrm{mag}$, respectively. The black line is a linear fit to the color indices. For the RG1 and RG2 samples, the two fitted black lines are parallel to each other (this is because the RG1 and RG2 samples are almost identical and they are computed using the same comparison band $J)$. The RCG samples are rarer and have a smaller range of $\left(J-K_{s}\right)$. The magenta dots plot all the sources with $\mathrm{S} / \mathrm{N} \geqslant 5$ in the RCG stripe in Figure 1(b), while the green ones are further limited to $K_{s} \leqslant 12$. In panel d, the fitted line derived from the RCG samples could be distorted by the contamination of YSOs and AGB stars. This is why we limit the $K_{s}$ magnitude to 12 for this GLIMPSE field. Gray dots plot all sources detected by GLIMPSE with S/N $\geqslant 5$ in all bands.

(A color version of this figure is available in the online journal.)

Table 2

Extinction (Relative to $A_{K_{s}}$ ) for the GLIMPSE Observation Strategy Validation (OSV) Field, with $283^{\circ} .8 \leqslant l \leqslant 284^{\circ} .6$ and $|b|<1^{\circ}$

\begin{tabular}{lcccc}
\hline \hline \multicolumn{1}{c}{ Tracers } & $A_{[3.6]} / A_{K_{S}}$ & $A_{[4.5]} / A_{K_{s}}$ & $A_{[5.8]} / A_{K_{s}}$ & $A_{[8.0]} / A_{K_{s}}$ \\
\hline Red giants (RG1, $J)$ & $0.609 \pm 0.002$ & $0.574 \pm 0.004$ & $0.478 \pm 0.004$ & $0.474 \pm 0.005$ \\
Red giants (RG2, $J$ ) & $0.612 \pm 0.002$ & $0.585 \pm 0.004$ & $0.487 \pm 0.004$ & $0.483 \pm 0.005$ \\
Red giants (RG2, $H$ ) & $0.578 \pm 0.002$ & $0.534 \pm 0.004$ & $0.429 \pm 0.005$ & $0.425 \pm 0.005$ \\
Red clump giants (RCG, $J)$ & $0.585 \pm 0.005$ & $0.436 \pm 0.006$ & $0.335 \pm 0.007$ & $0.40 \pm 0.01$ \\
Indebetouw05 $^{\mathrm{a}}$ & $0.57 \pm 0.05$ & $0.43 \pm 0.07$ & $0.41 \pm 0.07$ & $0.37 \pm 0.07$ \\
Flaherty07 & $0.57 \pm 0.01$ & $0.50 \pm 0.01$ & $0.40 \pm 0.01$ & $0.39 \pm 0.01$ \\
\hline
\end{tabular}

Note.

${ }^{\text {a }}$ Quoted values taken from Indebetouw et al. (2005) are for the off-cloud line of sight. They used red clump giants as tracers and the $J$ band as the comparison band, with $A_{J} / A_{K_{s}}=2.5 \pm 0.2$.

as theirs. The extinction values taken from Indebetouw et al. (2005) are for the off-cloud line of sight and derived from RCG stars. Although the extinction results are similar in the IRAC [3.6] and [4.5] bands, the extinction law derived here from the RCG samples for the OSV field is much steeper than that derived by Indebetouw et al. (2005), with much smaller values in the [5.8] band. This is probably due to different methods adopted in selecting RCG stars, such as the limitation on the $K_{s}$ magnitude or the criterion on color index (see Section 4.2.2). With the same criteria ([3.6] $-[4.5]<0.6$ and $[5.8]-[8.0]<0.2, \mathrm{RG} 2)$ and the 


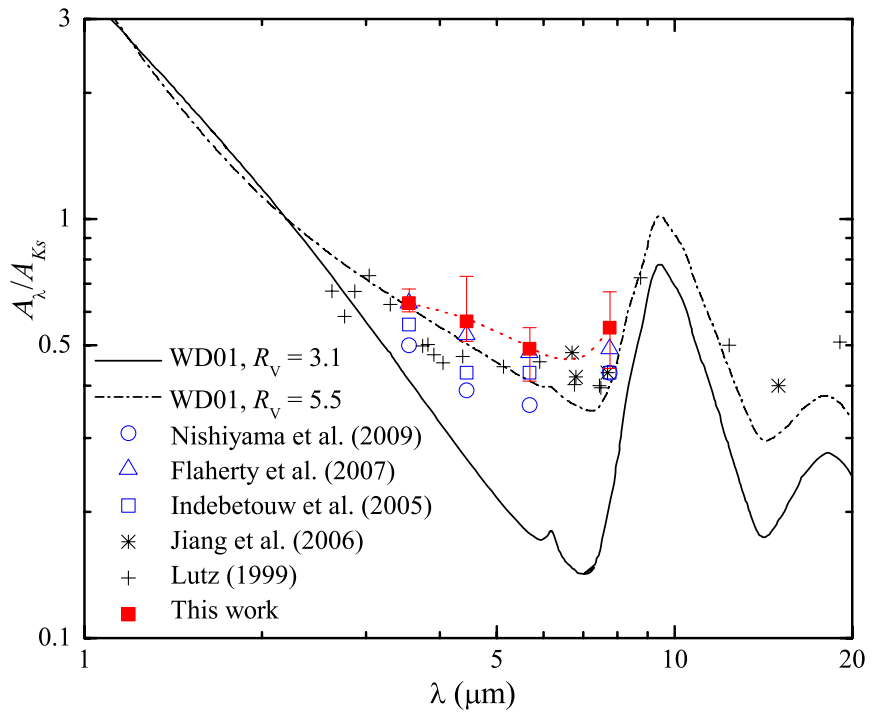

Figure 3. Comparison with previous observational determinations based on ISO and Spitzer data and with the extinction curves calculated from the interstellar grain model for $R_{V}=3.1$ (solid line) and for $R_{V}=5.5$ (dot-dashed line) of Weingartner \& Draine (2001; WD01). The dotted line smoothly connects the mean extinction ratios $A_{\lambda} / A_{K_{S}}$ derived in this work and that of Jiang et al. (2006) at $6.7 \mu \mathrm{m}$. The red bars plot the uncertainty ranges of $A_{\lambda} / A_{K_{s}}$ in each IRAC band.

(A color version of this figure is available in the online journal.)

same comparison band $H$ as adopted by Flaherty et al. (2007), our results are $\sim 0.3$ larger in the [4.5], [5.8], and [8.0] bands (see the third row of Table 2). The reason for this systematic difference could be attributed to the different power index $\beta$ used as discussed above. Nevertheless, the major reason why the extinction derived here (the first and second rows in Table 2) is systematically larger than that derived by Flaherty et al. (2007) for the OSV field lies in the choice of the comparison band: in this work, we use the $J$ band as the comparison band, while Flaherty et al. (2007) used the $H$ band.

In view of the different characteristics of RG and RCG stars, we averaged over both RG1 and RCG results in order to get a "mean" extinction law more or less unrelated to the distance. The "mean" interstellar extinction in the four IRAC bands are $\sim 0.63 \pm 0.01,0.57 \pm 0.03,0.49 \pm 0.03$, and $0.55 \pm 0.03$ for $A_{[3.6]} / A_{K_{s}}, A_{[4.5]} / A_{K_{s}}, A_{[5.8]} / A_{K_{s}}$, and $A_{[8.0]} / A_{K_{s}}$, respectively (see Table 1). In Figure 3, we plot our results together with previous observational determinations (Lutz 1999; Indebetouw et al. 2005; Jiang et al. 2006; Flaherty et al. 2007; Nishiyama et al. 2009) and the model extinction calculated for $R_{V}=3.1$ and $R_{V}=5.5$ by Weingartner $\&$ Draine (2001). It is seen in Figure 3 that our results are in close agreement with previous studies and confirm that the extinction law at $\sim 3-8 \mu \mathrm{m}$ is almost flat and lacks the minimum around $7 \mu \mathrm{m}$ predicted from the silicate-graphite interstellar grain model for $R_{V}=3.1$, but is close to (although systematically slightly higher than) the $R_{V}=5.5$ model curve.

\subsection{Variation of IR Extinction}

\subsubsection{Variation and Amplitude}

The interstellar extinction law is long known to vary along different sightlines in the optical and UV wavelength range, while the extinction in the near IR was often thought to be "universal" (but this was recently questioned by Nishiyama et al. 2006a). From the ISOGAL surveyed fields, Jiang et al. (2006) found marginal variation in $A_{7 \mu \mathrm{m}} / A_{K_{s}}$. Due to the relatively large uncertainty, the regional variation was not clear.

To examine whether there exist regional variations of the wavelength dependence of the IR extinction law, we show in Table 3 the extinction ratios $A_{\lambda} / A_{K_{s}}$ in all four IRAC bands for all 131 GLIMPSE fields, including results from both the RG1 and RCG samples. Because of more sample stars, much larger $E\left(J-K_{s}\right)$ and no limitation on the $K_{s}$ magnitude, the typical error for the RG1 results is just $\sim 0.001$, much smaller than that of the RCG results which is $\sim 0.01$. Figure 4 plots the histograms of the extinction ratios in all 131 GLIMPSE fields and clearly shows that there are a range of extinction ratios for both the RG1 and RCG results. The range for the RCG results is clearly larger than that for the RG1 results. Even for the extinction ratios derived from red giants, the range of variations for any of the IRAC bands is large enough to exceed the average error of $\sim 0.001$, with $\sim 0.07,0.16,0.14$, and 0.12 (see Table 4) at $3.6 \mu \mathrm{m}, 4.5 \mu \mathrm{m}, 5.8 \mu \mathrm{m}$, and $8.0 \mu \mathrm{m}$, respectively. This demonstrates that the extinction (relative to $A_{K_{s}}$ ) in the IRAC bands varies among different sightlines. A similar conclusion has already been drawn by Chapman et al. (2009), McClure (2009), Nishiyama et al. (2009), and Fitzpatrick \& Massa (2009).

The amplitude of the variation of $A_{\lambda} / A_{K_{s}}$ is relatively small for the $3.6 \mu \mathrm{m}$ band, and is thus difficult to detect without highquality data. This may explain why the extinction law in the near-IR was thought to be "universal" (Draine 2003). The variations in the $4.5 \mu \mathrm{m}$ and $5.8 \mu \mathrm{m}$ bands should be attributed to continuum extinction, while the variation of the $8 \mu \mathrm{m}$ extinction largely comes from the variation of the $9.7 \mu \mathrm{m}$ silicate absorption feature strength (see Figure 3) among different sightlines. The fact that the $8 \mu \mathrm{m}$ dispersion is essentially no greater than that of the $4.5 \mu \mathrm{m}$ and $5.8 \mu \mathrm{m}$ bands suggests that the $9.7 \mu \mathrm{m}$ silicate absorption feature probably does not vary much on these sightlines.

As shown in Figure 5, for the RG1 samples, $A_{[4.5]} / A_{K_{s}}$, $A_{[5.8]} / A_{K_{s}}$, and $A_{[8.0]} / A_{K_{s}}$ are all closely correlated with $A_{[3.6]} / A_{K_{s}}$, while the correlation between $A_{[8.0]} / A_{K_{s}}$ and $A_{[3.6]} / A_{K_{s}}$ for the RCG samples is not as tight as that of the [4.5] and [5.8] bands with the [3.6] band. This is probably due to the contamination of O-rich AGB stars at the lower end of RCG stripes: the extinction in the $3.6 \mu \mathrm{m}, 4.5 \mu \mathrm{m}$, and $5.8 \mu \mathrm{m}$ bands largely comes from the dust which produces the $\sim 1-6 \mu \mathrm{m}$ continuum extinction (e.g., graphite, see Figure 8 of Draine \& Lee 1984); in contrast, the $8.0 \mu \mathrm{m}$ extinction arises mainly from the $9.7 \mu \mathrm{m}$ silicate absorption feature. If the $3.6 \mu \mathrm{m}, 4.5 \mu \mathrm{m}$, and $5.8 \mu \mathrm{m}$ extinction is caused by interstellar dust, while the $8 \mu \mathrm{m}$ extinction is partly from interstellar dust and partly from O-rich AGB stars, one would expect that $A_{[8.0]} / A_{K_{s}}$ does not correlate with $A_{[3.6]} / A_{K_{s}}$ as closely as $A_{[4.5]} / A_{K_{s}}$ and $A_{[5.8]} / A_{K_{s}}$ correlate with $A_{[3.6]} / A_{K_{s}}$. We note that the absorption features of polycyclic aromatic hydrocarbon (PAH) at $6.2,7.7$, and $8.6 \mu \mathrm{m}$ are too weak to contribute to the discontinuities between RCG and RGs (see Figure 16 and Section 11 of Li \& Draine (2001), and Section 10.3 of Draine \& Li (2007).

\subsubsection{Variation with Galactic Longitude}

Unlike previous studies of the ISOGAL fields in which no clear structural distribution along Galactic longitude or latitude was found for the extinction at $7 \mu \mathrm{m}$ and $15 \mu \mathrm{m}$ (Jiang et al. 2006), in this work we see an interesting distribution of extinction. Whichever extinction tracer (red giants (RG1, RG2) or RCG stars) or sample-selection criterion is used, we always 
Table 3

Extinction Ratios $A_{\lambda} / A_{K_{s}}$ of the 131 GLIMPSE Fields

\begin{tabular}{|c|c|c|c|c|c|c|c|c|c|c|c|}
\hline \multirow[b]{2}{*}{ Field Name } & \multirow[b]{2}{*}{$l(\mathrm{deg})$} & \multirow[b]{2}{*}{$b(\mathrm{deg})$} & \multicolumn{4}{|c|}{ Red Giants (RG1) } & \multicolumn{5}{|c|}{ Red Clump Giants } \\
\hline & & & $A_{[3.6]} / A_{K_{s}}$ & $A_{[4.5]} / A_{K_{S}}$ & $A_{[5.8]} / A_{K_{s}}$ & $A_{[8.0]} / A_{K_{s}}$ & $A_{[3.6]} / A_{K_{s}}$ & $A_{[4.5]} / A_{K_{s}}$ & $A_{[5.8]} / A_{K_{S}}$ & $A_{[8.0]} / A_{K_{s}}$ & $\operatorname{Max}\left(K_{s}\right)$ \\
\hline GLMIIC_1000 & {$[0,1]$} & {$[-2,+2]$} & $0.651 \pm 0.001$ & $0.630 \pm 0.001$ & $0.541 \pm 0.001$ & $0.550 \pm 0.001$ & $0.70 \pm 0.01$ & $0.83 \pm 0.02$ & $0.56 \pm 0.02$ & $0.78 \pm 0.02$ & 11.5 \\
\hline GLMIC_1011 & {$[11,12]$} & {$[-1,+1]$} & $0.671 \pm 0.001$ & $0.669 \pm 0.001$ & $0.552 \pm 0.001$ & $0.587 \pm 0.001$ & $0.58 \pm 0.01$ & $0.46 \pm 0.01$ & $0.43 \pm 0.01$ & $0.56 \pm 0.01$ & 11.5 \\
\hline GLMIC_1019 & {$[19,20]$} & {$[-1,+1]$} & $0.653 \pm 0.001$ & $0.634 \pm 0.001$ & $0.532 \pm 0.001$ & $0.568 \pm 0.001$ & $0.62 \pm 0.01$ & $0.56 \pm 0.01$ & $0.50 \pm 0.01$ & $0.60 \pm 0.01$ & 12.0 \\
\hline GLMIC_1027 & {$[27,28]$} & {$[-1,+1]$} & $0.656 \pm 0.001$ & $0.650 \pm 0.001$ & $0.544 \pm 0.001$ & $0.581 \pm 0.001$ & $0.64 \pm 0.01$ & $0.58 \pm 0.01$ & $0.51 \pm 0.01$ & $0.64 \pm 0.01$ & 12.0 \\
\hline GLMIC_1035 & {$[35,36]$} & {$[-1,+1]$} & $0.643 \pm 0.001$ & $0.634 \pm 0.001$ & $0.526 \pm 0.001$ & $0.536 \pm 0.001$ & $0.60 \pm 0.01$ & $0.48 \pm 0.01$ & $0.42 \pm 0.01$ & $0.51 \pm 0.01$ & 12.0 \\
\hline GLMIC_1043 & {$[43,44]$} & {$[-1,+1]$} & $0.628 \pm 0.001$ & $0.598 \pm 0.001$ & $0.499 \pm 0.001$ & $0.514 \pm 0.001$ & $0.59 \pm 0.01$ & $0.50 \pm 0.01$ & $0.44 \pm 0.01$ & $0.53 \pm 0.01$ & 12.0 \\
\hline GLMIC_1051 & {$[51,52]$} & {$[-1,+1]$} & $0.640 \pm 0.001$ & $0.621 \pm 0.001$ & $0.523 \pm 0.001$ & $0.533 \pm 0.001$ & $0.60 \pm 0.01$ & $0.47 \pm 0.01$ & $0.40 \pm 0.01$ & $0.48 \pm 0.01$ & 12.0 \\
\hline GLMIC_1059 & {$[59,60]$} & {$[-1,+1]$} & $0.644 \pm 0.001$ & $0.633 \pm 0.002$ & $0.543 \pm 0.002$ & $0.548 \pm 0.002$ & $0.60 \pm 0.01$ & $0.51 \pm 0.01$ & $0.45 \pm 0.01$ & $0.51 \pm 0.01$ & 12.0 \\
\hline GLMIC_1295 & {$[295,296]$} & {$[-1,+1]$} & $0.646 \pm 0.002$ & $0.662 \pm 0.004$ & $0.568 \pm 0.004$ & $0.548 \pm 0.004$ & $0.62 \pm 0.01$ & $0.53 \pm 0.01$ & $0.37 \pm 0.01$ & $0.49 \pm 0.01$ & 12.5 \\
\hline GLMIC_1301 & {$[301,302]$} & {$[-1,+1]$} & $0.662 \pm 0.001$ & $0.656 \pm 0.002$ & $0.554 \pm 0.002$ & $0.560 \pm 0.002$ & $0.59 \pm 0.01$ & $0.48 \pm 0.01$ & $0.42 \pm 0.01$ & $0.50 \pm 0.01$ & 12.0 \\
\hline GLMIC_1309 & {$[309,310]$} & {$[-1,+1]$} & $0.640 \pm 0.001$ & $0.616 \pm 0.001$ & $0.520 \pm 0.001$ & $0.538 \pm 0.001$ & $0.60 \pm 0.01$ & $0.48 \pm 0.01$ & $0.41 \pm 0.01$ & $0.51 \pm 0.01$ & 12.0 \\
\hline GLMIC_1319 & {$[319,320]$} & {$[-1,+1]$} & $0.655 \pm 0.001$ & $0.666 \pm 0.002$ & $0.546 \pm 0.001$ & $0.563 \pm 0.002$ & $0.61 \pm 0.01$ & $0.49 \pm 0.01$ & $0.44 \pm 0.01$ & $0.54 \pm 0.01$ & 12.0 \\
\hline GLMIC_1327 & {$[327,328]$} & {$[-1,+1]$} & $0.633 \pm 0.001$ & $0.604 \pm 0.001$ & $0.508 \pm 0.001$ & $0.534 \pm 0.001$ & $0.61 \pm 0.01$ & $0.50 \pm 0.01$ & $0.42 \pm 0.01$ & $0.56 \pm 0.01$ & 12.0 \\
\hline GLMIC_1335 & {$[335,336]$} & {$[-1,+1]$} & $0.648 \pm 0.001$ & $0.639 \pm 0.001$ & $0.531 \pm 0.001$ & $0.558 \pm 0.001$ & $0.61 \pm 0.01$ & $0.49 \pm 0.01$ & $0.43 \pm 0.01$ & $0.56 \pm 0.01$ & 12.0 \\
\hline GLMIC_1343 & {$[343,344]$} & {$[-1,+1]$} & $0.639 \pm 0.001$ & $0.622 \pm 0.001$ & $0.516 \pm 0.001$ & $0.548 \pm 0.001$ & $0.60 \pm 0.01$ & $0.47 \pm 0.01$ & $0.40 \pm 0.01$ & $0.52 \pm 0.01$ & 12.0 \\
\hline
\end{tabular}

(This table is available in its entirety in a machine-readable form in the online journal. A portion is shown here for guidance regarding its form and content.) 


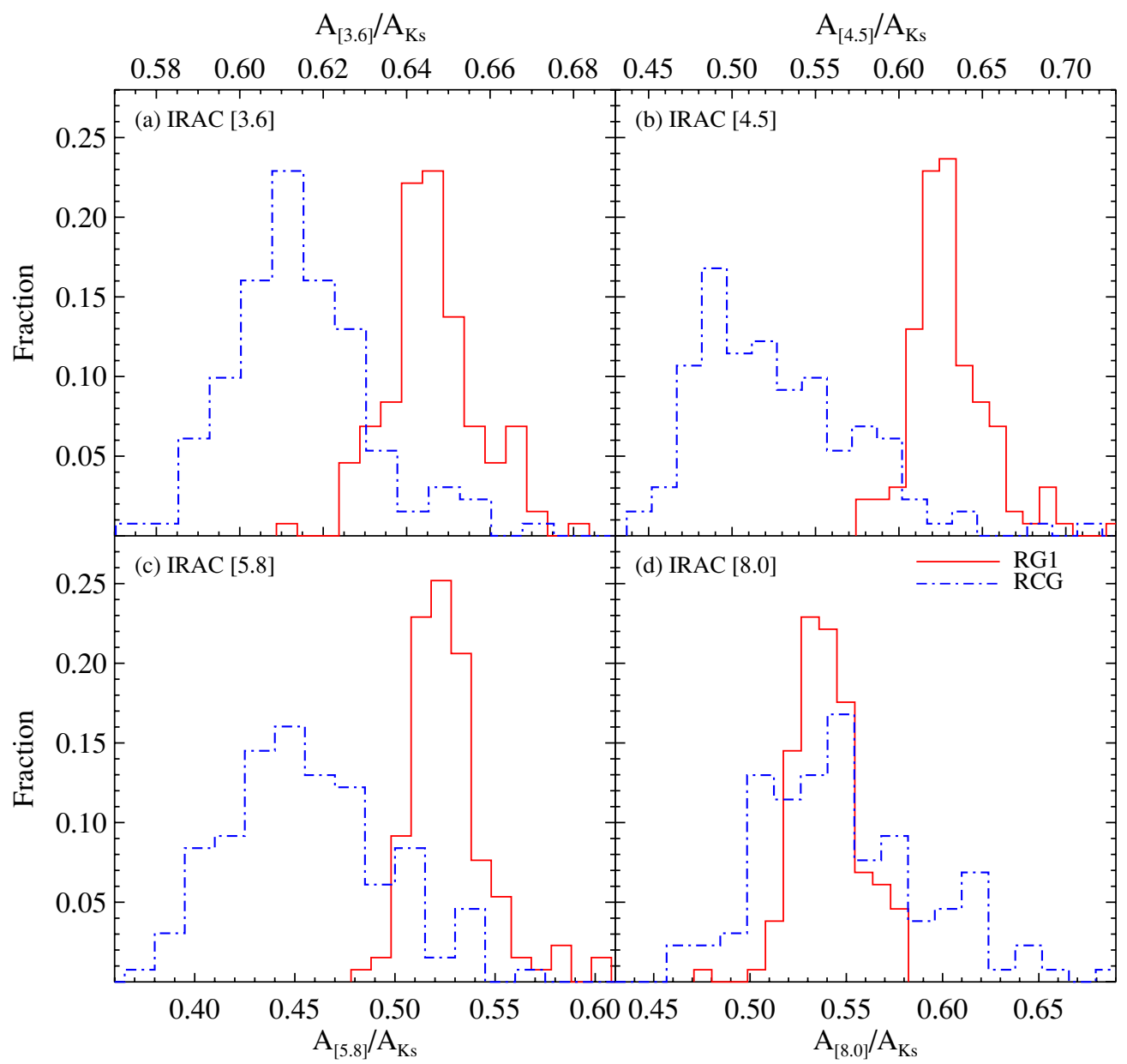

Figure 4. Histograms of the extinction (relative to $A_{K_{s}}$ ) of all 131 GLIMPSE fields in the four IRAC bands.

(A color version of this figure is available in the online journal.)

Table 4

Dispersion Ranges of the Extinction Ratios $A_{\lambda} / A_{K_{s}}$ Derived from Red Giants (RG1)

\begin{tabular}{lccc}
\hline \hline$\lambda(\mu \mathrm{m})$ & Minimum & Maximum & Range \\
\hline $3.6(3.545)$ & 0.61 & 0.68 & 0.07 \\
$4.5(4.442)$ & 0.57 & 0.73 & 0.16 \\
$5.8(5.675)$ & 0.48 & 0.62 & 0.14 \\
$8.0(7.760)$ & 0.47 & 0.59 & 0.12 \\
$7.0^{\mathrm{a}}$ & 0.37 & 0.55 & 0.18 \\
15.0 & 0.19 & 0.54 & 0.35 \\
\hline
\end{tabular}

Note.

${ }^{\text {a }}$ The $7 \mu \mathrm{m}$ and $15 \mu \mathrm{m}$ extinction data are taken from Jiang et al. (2006).

see an uneven distribution of extinction ratios in each IRAC band with Galactic longitude (see Figure 6). Since the results from the RG1 and RG2 samples are approximately the same, we only show that of the RG1 (and RCG) samples in Figure 6.

Longitudinal extinction profiles derived from RG1 or RCG samples. Although the extinction values derived from the RCG stars are systematically smaller than that from the red giants as discussed in the previous section, the structural features appear at almost the same positions outside the Galactic bulge region for both samples. The common features include, dips around $l=-50^{\circ}, l=-20^{\circ}, l=16^{\circ}, l=51^{\circ}$, peaks around $l=-53^{\circ}$ (see Figure 6). However, in the bulge direction within $|l|<15^{\circ}$, the two samples yield different variation patterns. This can be understood in view of the fact that the RCG samples selected in this work do not trace as deeply as the red giant samples to the Galactic bulge due to the limiting magnitude of $K_{s}<11.5$ on the RCG stars in these regions. As mentioned earlier, the selected RCG samples can only reach a distance of $\sim 4 \mathrm{kpc}$ from us to the bulge direction even if there is no extinction. In contrast, the red giants trace the structure of the bulge such as the bar and the distant spiral arms, which shows some of the differences in the longitudinal distribution of $A_{\lambda} / A_{K_{s}}$ toward the GC. Another difference between the results from the RG1 and RCG samples lies in the regions between $20^{\circ}<l<30^{\circ}$. Similarly, the RCG samples can only probe the interstellar extinction of the ISM of nearby spiral arms (e.g., the Sagittarius-Carina arm located at $\sim 2 \mathrm{kpc}$ from the Sun), but not the extinction from the more distant $\sim 3 \mathrm{kpc}$-molecular ring between $20^{\circ}<l<30^{\circ}$ which contains a significant amount of $\mathrm{CO}$ and dust (Hammersley et al. 1994; Dame et al. 2001; Dame \& Thaddeus 2008). ${ }^{20}$ Meanwhile, the RCG stars can only trace the Crux-Scutum arm at $l \approx-29^{\circ}$ (Hammersley et al. 1994), not the more distant Norma arm (Vallée 2008); thus, the longitudinal profiles from RCGs and RGs are also different along this direction.

\footnotetext{
20 van Woerden et al. (1957) suggested the apparent southern tangent for the near $3 \mathrm{kpc}$ ring is at $l=-22^{\circ}$. Cohen et al. (1980) suggested the northern tangent is near $l=24^{\circ}$. Dame \& Thaddeus (2008) adopted tangent directions of $\pm 23^{\circ}$ for the $3 \mathrm{kpc}$ arm based on their composite CO survey. With $R_{\odot}=8.5 \mathrm{kpc}$, the near arm is at a distance of $\sim 5.2 \mathrm{kpc}$ (Dame \& Thaddeus 2008).
} 


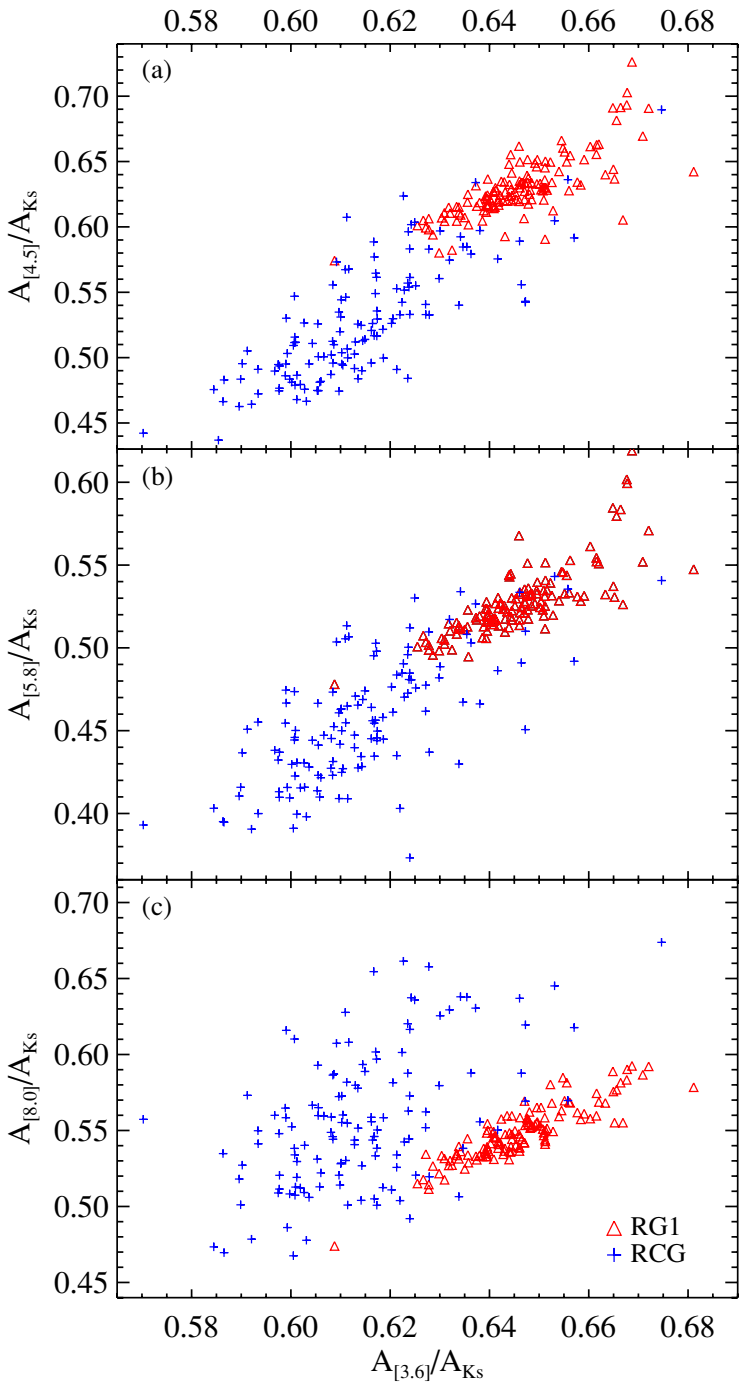

Figure 5. Correlation of $A_{[4.5]} / A_{K_{s}}, A_{[5.8]} / A_{K_{s}}$, and $A_{[8.0]} / A_{K_{s}}$ with $A_{[3.6]} / A_{K_{s}}$. Red, unfilled triangles are for RG1; blue crosses are for RCG.

(A color version of this figure is available in the online journal.)

Relationship with the Galactic spiral arms. Because the dust properties of the spiral arm regions differ from those of the interarm regions (Greenberg \& $\mathrm{Li}$ 1995), the variation of extinction with Galactic longitude could be related to the spiral structure. We label the tangent positions of the spiral arms in Figure 6 with long black arrows. ${ }^{21}$ It is seen that the locations of the spiral arms coincide with the dips of the extinction ratios $A_{\lambda} / A_{K_{s}}$ derived from the RG1 samples. ${ }^{22}$ In particular,

\footnotetext{
21 The number of spiral arms and other arm parameters (e.g., pitch angles) have not been well determined, although there have been various investigations to determine them. For example, the number of spiral arms (two or four) is actively being debated (e.g., see Benjamin 2008). We favor the four-arm model for the sake of studying the dust extinction profile versus the Galactic structure since the $240 \mu \mathrm{m}$ dust emission supports the four-arm model (Drimmel 2000). Note that both the extinction and $240 \mu \mathrm{m}$ emission are from dust. Vallée (2008) evaluated, compiled and compared previous works on the determination of the position parameters of the spiral arms. In his four-arm structure model, Vallée (2008) placed the mean longitudes of tangent from the Sun to the Carina-Sagittarius arm, the Crux-Scutum arm, the Norma arm, the start of the Perseus arm, the Scutum-Crux arm, and the Sagittarius-Carina arm at $l=-76^{\circ} \pm 2^{\circ},-50^{\circ} \pm 3^{\circ},-33^{\circ} \pm 3^{\circ},-21^{\circ} \pm 2^{\circ}, 31^{\circ} \pm 3^{\circ}$ and $51^{\circ} \pm 4^{\circ}$, respectively (see his Table 2 and Figure 2).

22 Since the RCG samples cannot probe the extinction to distant arms (therefore they cannot reflect the large-scale structure of the spiral arms), we will only consider $A_{\lambda} / A_{K_{s}}$ from the RG1 samples in the following discussion.
}

the coincidence of the locations of the $A_{\lambda} / A_{K_{s}}$ minimum, and the spiral arms is outstanding at negative longitudes (e.g., the Crux-Scutum arm at $l=-50^{\circ}$, the Norma arm at $-33^{\circ}$, and the southern tangent of the $3 \mathrm{kpc}$ ring around $-23^{\circ}$ ). For the positive longitudes, the locations of the Scutum-Crux arm and the Sagittarius-Carina arm also coincide with the valleys of $A_{\lambda} / A_{K_{\mathrm{s}}}$. For the broad dip around $l=16^{\circ}$, there is no clear tangent direction to any arms, but this direction points to the start of the Norma arm and the Scutum-Crux arm (see Figure 2 of Vallée 2008), and probably one end of the Galactic bar. ${ }^{23}$ Hammersley et al. (1994) found an extra dip around $l=16^{\circ}$ in the longitude distribution of the $2.2 \mu \mathrm{m}$ flux obtained by the Diffuse Infrared Background Experiment (DIRBE) on board the Cosmic Background Explorer (COBE, see their Figure 1). They speculated that it results from a large dust cloud in a spiral arm or a molecular ring. In addition, they also found a broad $2.2 \mu \mathrm{m}$ valley between about $l=22^{\circ}$ and $26^{\circ}$, which corresponds to the expected position of the $3 \mathrm{kpc}$ ring. Again, the dip of $A_{\lambda} / A_{K_{s}}$ around $l=25^{\circ}$ may be consistent with the location of the $3 \mathrm{kpc}$ ring.

The dip of the longitudinal distribution of $A_{\lambda} / A_{K_{s}}$ at $l \simeq$ $-30^{\circ}$ appears shifted from the position of the Norma arm at $l=-33^{\circ}$. But we note that the longitudinal position of the tangent to the Norma arm is not precisely known and the reported positions range from $l=-37^{\circ}$ to $-28^{\circ}$ (Vallée 2008; e.g., $l=-31^{\circ}$, Bloemen et al. 1990; $l=-29^{\circ}$, Hammersley et al. 1994; $l=-32^{\circ}$, Bronfman 2008). This probably also explains why the dip of the longitudinal distribution of $A_{\lambda} / A_{K_{s}}$ at $l=48^{\circ}$ does not precisely match the tangent direction to the Sagittarius-Carina arm at " $l=50^{\circ}$ " (for which the reported positions range from $l=46^{\circ}$ to $56^{\circ}$, Vallée 2008).

Why does $A_{\lambda} / A_{K_{s}}$ reach its minimum in the Galactic spiral arm regions? A simple explanation would be grain growth: in spiral galaxies, interstellar gas and dust are concentrated in the inner edges of spiral arms (probably caused by the spiral density wave; Greenberg 1970). Greenberg \& Li (1995) argued that as the interarm matter encounters the density wave potential minimum, the gas is compressed by the density wave shock (with a speed of a few tens $\mathrm{km} \mathrm{s}^{-1}$; Roberts 1969; Roberts et al. $1975,1979)$ at the inner edge of the spiral arm. This would lead not only to a larger number density of gas and dust, but also an increase of grain size by accretion and coagulation. As shown in Figure 7, if the dust in the spiral arm regions (where giant molecular clouds are strongly concentrated) grows to $a \sim 0.2-0.3 \mu \mathrm{m}$, the wavelength dependence of $A_{\lambda} / A_{K_{s}}$ in the wavelength range of $2-8 \mu \mathrm{m}$ becomes steeper (i.e., smaller) than that of dust of radii $a=0.1 \mu \mathrm{m}$ (mean size for the dust in the diffuse ISM) for amorphous silicate, amorphous carbon, and graphite. This explains why the $A_{\lambda} / A_{K_{s}}$ values are small in the Galactic spiral arms for all four IRAC bands.

Relationship with the dust IR emission. In Figure 8, we plot the longitudinal distribution of the Galactic $240 \mu \mathrm{m}$ emission from the DIRBE Galactic Plane Maps ${ }^{24}$ with $A_{[3.6]} / A_{K_{s}}$, as well as the locations of the spiral arms (Vallée 2008). We see that most of the dips of the $A_{[3.6]} / A_{K_{s}}$ profile coincide with the peaks of the $240 \mu \mathrm{m}$ emission (e.g., the features around $\left.l=-55^{\circ},-49^{\circ},-29^{\circ},-23^{\circ},-8^{\circ}, 25^{\circ}, 31^{\circ}, 38^{\circ}, 49^{\circ}\right)$.

\footnotetext{
${ }^{23}$ In Vallee's model, the Galactic bar is simply drawn on his cartographic model with the mean values from the literature. Benjamin et al. (2005) determined the radius $\left(R_{\mathrm{bar}}=4.4 \pm 0.5 \mathrm{kpc}\right)$ and orientation $\left(\phi=44^{\circ} \pm 10^{\circ}\right)$ of the Galactic bar from the M and $\mathrm{K}$ giants in the GLIMPSE survey. ${ }^{24}$ The data are available on http://lambda.gsfc.nasa.gov/product/cobe/ dirbe_gpm_data_get.cfm.
} 


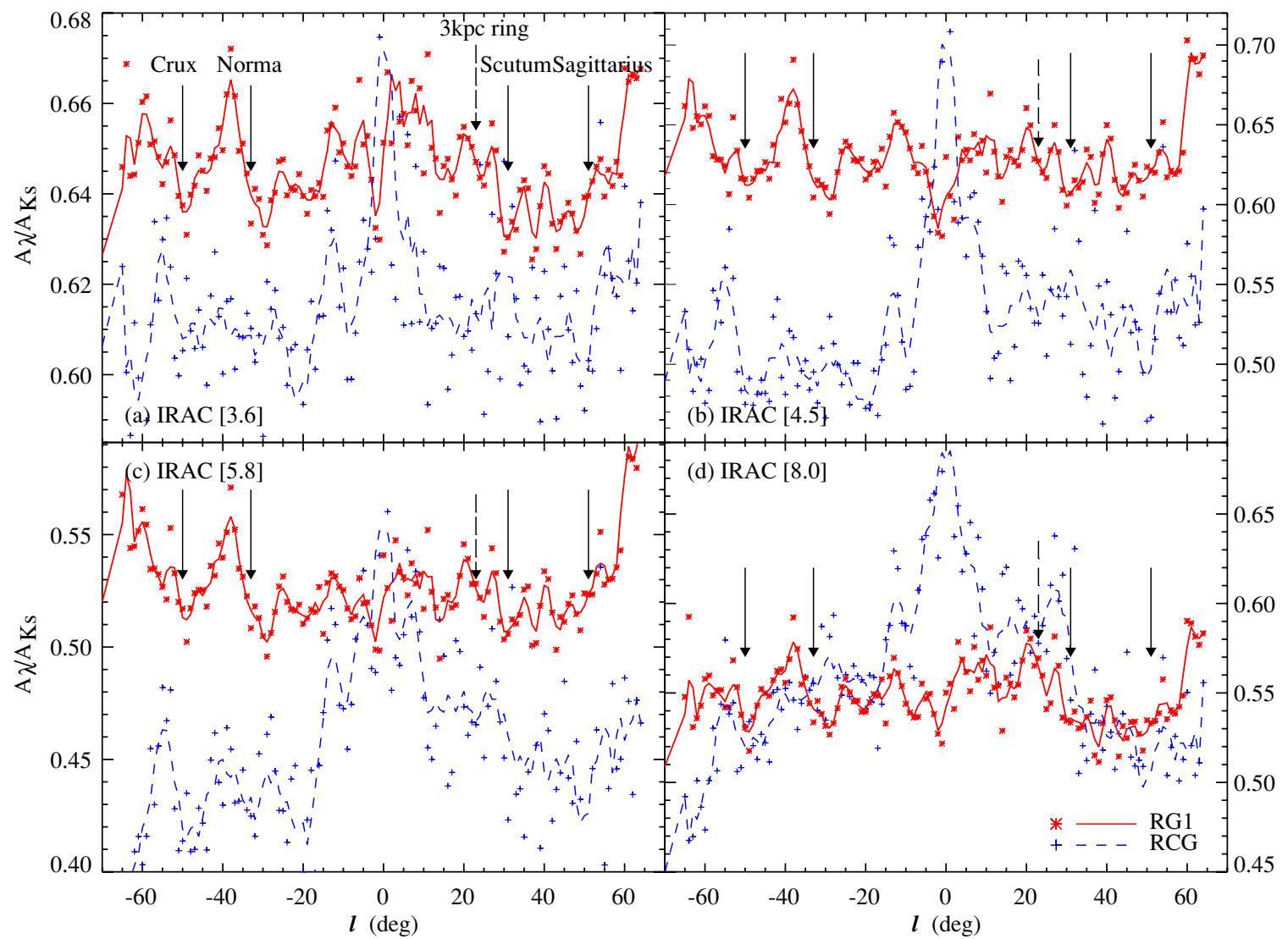

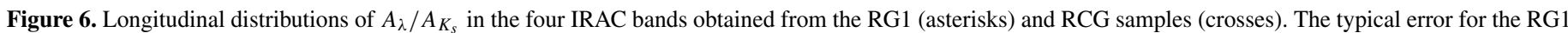

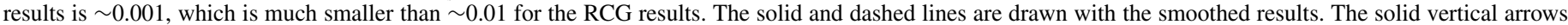

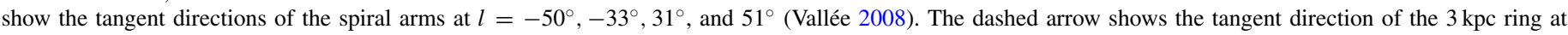
$l=23^{\circ}$ (Dame \& Thaddeus 2008).

(A color version of this figure is available in the online journal.)

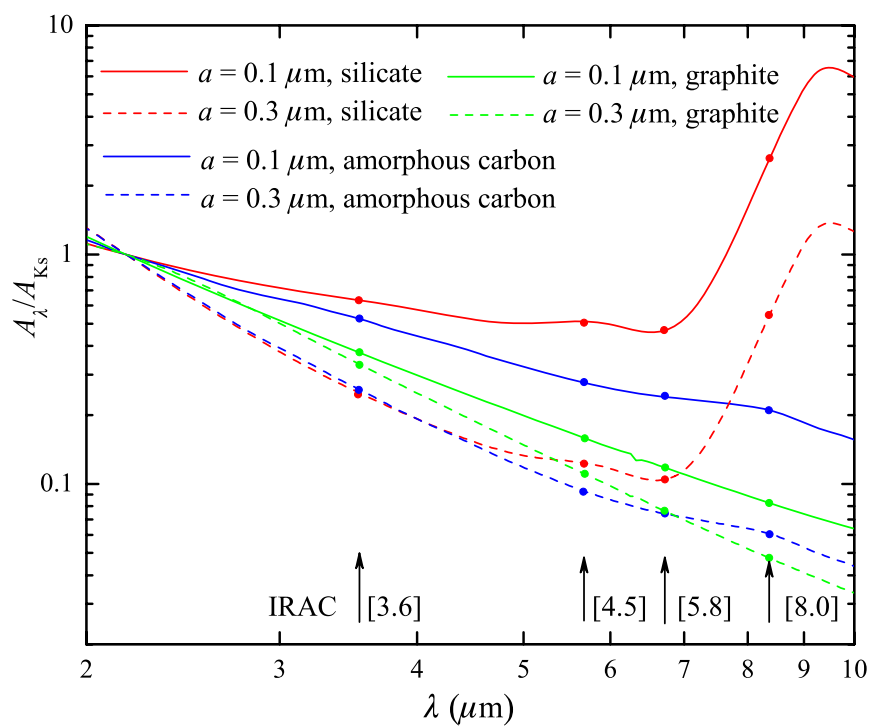

Figure 7. Wavelength dependence of extinction (normalized in the $K_{s}$ band) $A_{\lambda} / A_{K_{s}}$ of spherical grains of radii $a=0.1 \mu \mathrm{m}$ and $0.3 \mu \mathrm{m}$ of amorphous silicate, graphite, or amorphous carbon composition. The $A_{\lambda} / A_{K_{S}}$ values in four IRAC bands are marked with filled circles. We see that if the dust in the Galactic spiral arm regions grows to $a \sim 0.3 \mu \mathrm{m}$, one would expect a steeper extinction law (i.e., smaller $A_{\lambda} / A_{K_{s}}$ ratios) than that of the diffuse ISM for which the mean dust size is $\sim 0.1 \mu \mathrm{m}$.

(A color version of this figure is available in the online journal.)
Drimmel (2000) found that the features in the $240 \mu \mathrm{m}$ emission profile from dust can be identified with spiral arm tangents. Similarly, most of the dips of the extinction profile can be understood in terms of the locations of the spiral arms (e.g., the $240 \mu \mathrm{m}$ emission peak and the $A_{[3.6]} / A_{K_{s}}$ dip at $l=-49^{\circ}$ versus the Crux-Scutum arm, the $l=-29^{\circ}$ feature versus the Norma arm, the $l=31^{\circ}$ feature versus the Scutum-Crux arm, the $l=49^{\circ}$ feature versus the Sagittarius-Carina arm; the features around $l=-23^{\circ}$ and $l=25^{\circ}$ versus the $3 \mathrm{kpc}$ ring or arm): in the spiral arm regions which are rich in star-forming giant molecular clouds and $\mathrm{H}$ II regions, not only is the dust size relatively larger because of accretion and coagulational growth (which explains the coincidence of the dips of $A_{\lambda} / A_{K_{s}}$ with the locations of the spiral arms), but the dust number density is also higher because of the gas and dust concentration in the inner edges of spiral arms caused by the spiral density wave, and the starlight intensity which illuminates the dust causing it to emit at $240 \mu \mathrm{m}$ is also higher because of the star formation activity occurring in the arm. Both the increased dust concentration and starlight intensity would result in a higher $240 \mu \mathrm{m}$ emission.

The Galactic bulge region. In all four IRAC bands, the extinction ratios $A_{\lambda} / A_{K_{s}}$ derived from red giants decrease for the sightlines toward the bulge region and reach their minimum values near $l=0^{\circ}$, while it is the opposite for that derived from the RCG samples. This difference is probably due to the limited distance which the RCG samples can probe, as discussed earlier in Section 4.2.2. In addition, the extinction is not simply a linear function of the distance because of the nonuniform distribution 


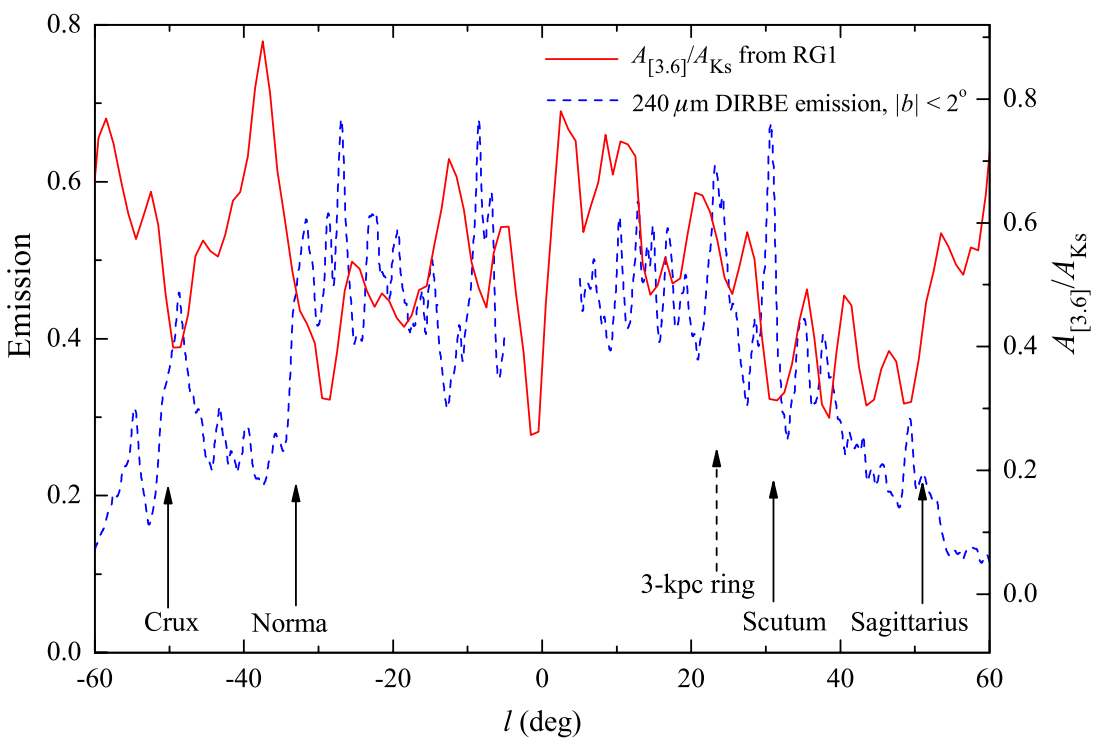

Figure 8. Longitudinal profiles of $A_{[3.6]} / A_{K_{s}}$ and the $240 \mu \mathrm{m}$ dust emission from the DIRBE Galactic Plane Maps (averaged over the latitude interval $|b|<2^{\circ}$ ). Most of the dips on the $A_{[3.6]} / A_{K_{s}}$ profile coincide with the $240 \mu \mathrm{m}$ emission peak.

(A color version of this figure is available in the online journal.)

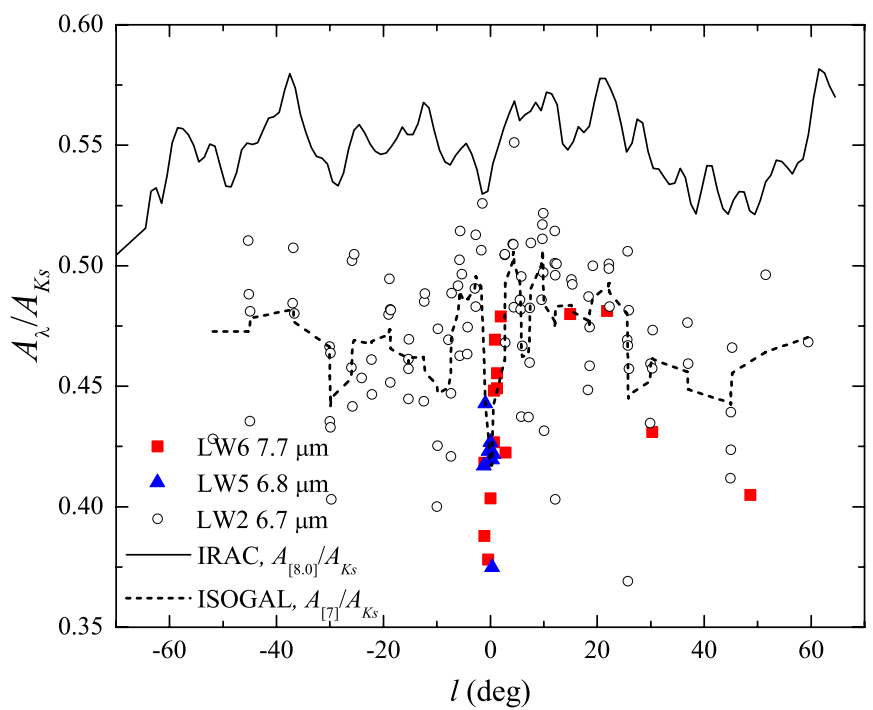

Figure 9. Longitudinal profiles of $A_{[8.0]} / A_{K_{s}}$ (solid line) and $A_{[7]} / A_{K_{s}}$ (dashed line). The latter was obtained from smoothing, re-analyzing the ISO LW2 $(6.7 \mu \mathrm{m})$, LW5 $(6.8 \mu \mathrm{m})$ and LW6 $(7.7 \mu \mathrm{m})$ data of the ISOGAL fields (Jiang et al. 2006).

(A color version of this figure is available in the online journal.)

(i.e., grain density, size, and composition) of interstellar dust along these directions. ${ }^{25}$ Thus, the extinction probed by red

\footnotetext{
${ }^{25}$ For example, it has been observationally demonstrated that both the ratio of the visual extinction $\left(A_{V}\right)$ to the $9.7 \mu \mathrm{m} \mathrm{Si}-\mathrm{O}$ optical depth $\left(\Delta \tau_{9.7 \mu \mathrm{m}}\right)$ and the ratio of $A_{V}$ to the $3.4 \mu \mathrm{m} \mathrm{C}-\mathrm{H}$ optical depth $\left(\Delta \tau_{3.4 \mu \mathrm{m}}\right)$ show considerable variations from the local diffuse ISM to the GC (see Gao et al. 2009). Sandford et al. (1995) explained this by assuming that the abundance of the $\mathrm{C}-\mathrm{H}$ carrier (relative to other dust components) gradually increases from the local ISM toward the GC. Using the RCG stars in the GC within $|l|<2^{\circ}$ as a probe, Nishiyama et al. (2006a) found that the interstellar extinction of these areas is indeed highly nonuniform. Also the conventional linear relation between extinction and distance of $A_{V} / d \approx 1.8 \mathrm{mag} \mathrm{kpc}^{-1}$ was obtained for stars within $\sim 1000 \mathrm{pc}$ of the Sun and within $\sim 100 \mathrm{pc}$ of the Galactic plane (Spitzer 1978). Toward the galactic bulge, in view of the large-scale structure of the ISM, the small-scale patchy distribution of dust seems well established (see Spitzer 1978)
}

giants and RCG stars in this work may be very different for the sightlines toward the bulge region.

The decreasing trend of $A_{\lambda} / A_{K_{s}}$ toward the GC suggests that the extinction law toward the GC may be different from that of the local diffuse ISM or star-forming regions. Recently, using the bulge RCG stars, Nishiyama et al. (2009) obtained the $\sim 1.2-$ $8 \mu \mathrm{m}$ interstellar extinction law toward the GC. They found an appreciably steeper extinction law compared with that of the offcloud regions in the Galactic plane of Indebetouw et al. (2005) and the star-forming regions of Flaherty et al. (2007).

Jiang et al. (2006) studied the ISOGAL fields using red giants and found no clear structural distribution of the $7 \mu \mathrm{m}$ extinction along Galactic longitude. We re-analyzed the extinction around $7 \mu \mathrm{m}$ by smoothing the distribution through a 3 -degree bin. The results (based on the ISO/SWS $6.7 \mu \mathrm{m}$ LW2, $6.8 \mu \mathrm{m}$ LW5, and $7.7 \mu \mathrm{m}$ LW6 data) are shown in Figure 9. The smoothed, re-analyzed data appear to display a longitude variation of $A_{[7 \mu \mathrm{m}]} / A_{K_{s}}$, with the local minimum at the GC as the most notable feature. In particular, the extinction ratio $A_{7.7 \mu \mathrm{m}} / A_{K_{s}}$ shows a profile similar to that derived from the RG1 samples.

\section{CONCLUSIONS}

Using red giants and red clump giants as tracers, we have derived $A_{\lambda} / A_{K_{s}}$, the extinction (relative to the 2MASS $K_{s}$ band) in the four IRAC bands, [3.6], [4.5], [5.8] and [8.0] $\mu \mathrm{m}$ for 131 GLIPMSE fields along the Galactic plane within $|l| \leqslant 65^{\circ}$, based on the data obtained from the Spitzer/GLIPMSE Legacy Program and the 2MASS Survey project. The principal results of this paper are as follows.

1. The mean extinction in the IRAC bands (normalized to the 2MASS $K_{s}$ band), $A_{[3.6]} / A_{K_{\mathrm{s}}} \approx 0.63 \pm 0.01, A_{[4.5]} / A_{K_{\mathrm{s}}} \approx$ $0.57 \pm 0.03, A_{[5.8]} / A_{K_{s}} \approx 0.49 \pm 0.01$, and $A_{[8.0]} / A_{K_{s}} \approx$ $0.55 \pm 0.03$, exhibits little variation with wavelength and lacks the minimum at $\sim 7 \mu \mathrm{m}$ predicted from the standard interstellar grain model for $R_{V}=3.1$. This is consistent within errors with previous observational determinations based on ISO and Spitzer data and with that predicted from the grain model for $R_{V}=5.5$ of Weingartner \& Draine (2001). 
2. The wavelength dependence of interstellar extinction in the mid-IR varies from one sightline to another, suggesting that there may not exist a "universal" IR extinction law.

3. There exist systematic variations of extinction with Galactic longitude which appears to correlate with the locations of spiral arms and with the variation of the $240 \mu \mathrm{m}$ dust emission. This can be understood in terms of larger grain sizes (arising from coagulational growth), enhanced dust concentration, and higher starlight intensities in the spiral arm regions.

We thank the anonymous referee for his/her very helpful comments. We thank the referee and Dr. Robert Benjamin for providing us the extinction versus $\mathrm{CO}$ emission data. We thank Drs. Y.-Q. Lou and J.P. Vallée for fruitful discussions. This work is supported by the NSFC grants no. 10603001 and 10973004 , the grant 2007CB815406. A.L. is supported in part by the Spitzer Cycle 3 GO program P30403, the NASA/Herschel Cycle 0 Theory grant, and NSF grant AST 07-07866.

Facilities: Spitzer(IRAC), 2MASS.

\section{REFERENCES}

Alves, D. R. 2000, ApJ, 539, 732

Allen, L. E., et al. 2004, ApJS, 154, 363

Benjamin, R. A., et al. 2003, PASP, 115, 953

Benjamin, R. A., et al. 2005, ApJ, 630, L149

Benjamin, R. A. 2008, in ASP Conf. Ser. 387, Massive Star Formation: Observations Confront Theory, ed. H. Beuther, H. Linz, \& Th. Henning (San Francisco, CA: ASP), 375

Bertelli, G., Bressan, A., Chiosi, C., Fagotto, F., \& Nasi, E. 1994, A\&AS, 106, 275

Bloemen, J. B., Deul, E. R., \& Thaddeus, P. 1990, A\&A, 233, 437

Bronfman, L. 2008, Ap\&SS, 313, 81

Cabrera-Lavers, A., Hammersley, P. L., González-Fernández, C., LópezCorredoira, M., Garzón, F., \& Mahoney, T. J. 2007, A\&A, 465, 825

Cardelli, J. A., Clayton, G. C., \& Mathis, J. S. 1989, ApJ, 345, 245

Chapman, N. L., Mundy, L. G., Lai, S.-P., \& Evans, N. J. 2009, ApJ, 690, 496

Churchwell, E., et al. 2006, GLIMPSE Quality Assurance v1.0. (Madison, WI: Univ. Wisconsin), http://www.astro.wisc.edu/sirtf/GQA-master.pdf

Churchwell, E., et al. 2009, PASP, 121, 213

Cohen, R. S., Cong, H., Dame, T. M., \& Thaddeus, P. 1980, ApJ, 239, L53

Cutri, R. M., et al. 2003, The IRSA 2MASS All-Sky Point Source Catalog NASA/IPAC Infrared Science Archive (Pasadena, CA: CalTech), http://irsa.ipac.caltech.edu/applications/Gator/

Dame, T. M., Hartmann, D., \& Thaddeus, P. 2001, ApJ, 547, 792

Dame, T. M., \& Thaddeus, P. 2008, ApJ, 683, L143

Demers, S., Irwin, M. J., \& Gambu, I. 1994, MNRAS, 266, 7

Draine, B. T., \& Lee, H. M. 1984, ApJ, 285, 89

Draine, B. T. 1989, in Infrared Spectroscopy in Astronomy, ed. B. H. Kaldeich (Paris: ESA Publications Division), 93

Draine, B. T. 2003, ARA\&A, 41, 241

Draine, B. T., \& Li, A. 2007, ApJ, 657, 810

Drimmel, R. 2000, A\&A, 358, L13
Drimmel, R., Cabrera-Lavers, A., \& López-Corredoira, M. 2003, A\&A, 409, 205

Fitzpatrick, E. L. 2004, in ASP Conf. Ser. 309, Astrophysics of Dust, ed. A. N. Witt, G. C. Clayton, \& B. T. Draine (San Francisco, CA: ASP), 33

Fitzpatrick, E. L., \& Massa, D. 2009, ApJ, 699, 1209

Flaherty, K., Pipher, J., Megeath, S., Wisnton, E., Gutermuth, R., Muzerolle, J., Allen, L., \& Fazio, G. 2007, ApJ, 663, 1069

Gao, J., Jiang, B. W., \& Li, A. 2009, Earth, Planets \& Space, in press

Glass, I., et al. 1999, MNRAS, 308, 127

Greenberg, J. M. 1970, in IAU Symp. 39, Interstellar Gas Dynamics, ed. H. J. Habing (Dordrecht: Reidel), 306

Greenberg, J. M., \& Li, A. 1995, in NATO ASI Conf. Proc. 469, The Opacity of Spiral Disks, ed. J. I. Davies \& D. Burstein (Dordrecht: Kluwer), 19

Groenewegen, M. 2006, A\&A, 448, 181

Hammersley, P. L., Garzón, F., Mahoney, T. J., \& Calbet, X. 1994, MNRAS, 269,753

Hammersley, P. L., Garzón, F., Mahoney, T. J., López-Corredoira, M., \& Torres, M. A. P. 2000, MNRAS, 317, L45

Indebetouw, R., et al. 2005, ApJ, 619, 931

Jiang, B. W., Omont, A., Ganesh, S., Simon, G., \& Schuller, F. 2003, A\&A, 400, 903

Jiang, B. W., Gao, J., Omont, A., Schuller, F., \& Simon, G. 2006, A\&A, 446, 551

Li, A., \& Draine, B. T. 2001, ApJ, 554, 778

López-Corredoira, M., Cabrera-Lavers, A., Garzón, F., \& Hammersley, P. L. 2002, A\&A, 394, 883

Lutz, D., et al. 1996, A\&A, 315, L269

Lutz, D. 1999, in The Universe as Seen by ISO, ed. P. Cox \& M. F. Kessler (ESA SP-427; Noordwijk: ESA Publications Division), 623

Marengo, M., Hora, J. L., Barmby, P., Willner, S. P., Allen, L. E., Schuster, M. T., \& Fazio, G. G. 2007, in ASP Conf. Ser. 378, Why Galaxies Care About AGB Stars: Their Importance as Actors and Probes, ed. F. Kerschbaum, C. Charbonnel, \& R. F. Wing (San Francisco CA: ASP), 80

Marengo, M., Reiter, M., \& Fazio, G. G. 2008, Evolution and Nucleosynthesis in AGB Stars, 1001, 331

McClure, M. 2009, ApJ, 693, L81

Megeath, S. T., Gutermuth, R. A., Allen, L. E., Pipher, J. L., Myers, P. C., \& Fazio, G. G. 2004, ApJS, 154, 367

Nishiyama, S., et al. 2006a, ApJ, 638, 839

Nishiyama, S., et al. 2006b, ApJ, 647, 1093

Nishiyama, S., Tamura, M., Hatano, H., Kato, D., Tanabé, T., Sugitani, K., \& Nagata, T. 2009, ApJ, 696, 1407

Omont, A., et al. 1999, A\&A, 348, 755

Rieke, G., \& Lebofsky, M. 1985, ApJ, 288, 618

Roberts, W. W. 1969, ApJ, 158, 123

Roberts, W. W., Roberts, M. S., \& Shu, F. H. 1975, ApJ, 196, 381

Roberts, W. W., Jr., Huntley, J. M., \& van Albada, G. D. 1979, ApJ, 233, 67

Román-Zúñiga, C. G., Lada, C. J., Muench, A., \& Alves, J. F. 2007, ApJ, 664, 357

Sandford, S. A., Pendleton, Y. J., \& Allamandola, L. J. 1995, ApJ, 440, 697

Spitzer, L. 1978, Physical Processes in the Interstellar Medium (New York: Wiley Interscience), 46

Vallée, J. P. 2008, AJ, 135, 1301

van Loon, J. T., et al. 2003, MNRAS, 338, 857

van Woerden, H., Rougoor, G. W., \& Oort, J. H. 1957, C. R. Acad. Sci., 244, 1691

Vassiliadis, E., \& Wood, P. R. 1993, ApJ, 413, 641

Wainscoat, R., Cohen, M., Volk, K., Walker, H., \& Schwartz, D. 1992, ApJS, 83,146

Weingartner, J. C., \& Draine, B. T. 2001, ApJ, 548, 296

Whittet, D. C. B. 1977, MNRAS, 180, 29 